\title{
The structure of planetary nebulae: theory vs. practice ${ }^{\star, \star \star}$
}

\author{
F. Sabbadin ${ }^{1}$, M. Turatto ${ }^{1}$, R. Ragazzoni ${ }^{2}$, E. Cappellaro ${ }^{3}$, and S. Benetti ${ }^{1}$ \\ 1 INAF - Astronomical Observatory of Padua, Vicolo dell'Osservatorio 5, 35122 Padua, Italy \\ e-mail: sabbadin@pd.astro.it \\ 2 INAF - Astrophysical Observatory of Arcetri, Largo E. Fermi 5, 50125, Italy \\ INAF - Astronomical Observatory of Capodimonte, via Moiariello 11, 80131 Naples, Italy
}

Received 21 November 2005 / Accepted 12 January 2006

\begin{abstract}
Context. This paper is the first in a short series dedicated to the long-standing astronomical problem of de-projecting the bidimensional, apparent morphology of a three-dimensional mass of gas.

Aims. We focus on the density distribution in real planetary nebulae (and all types of expanding nebulae).

Methods. We introduce some basic theoretical notions, discuss the observational methodology, and develop an accurate procedure for determining the matter radial profile within the sharp portion of nebula in the plane of the sky identified by the zero-velocity-pixelcolumn (zvpc) of high-resolution spectral images.

Results. The general and specific applications of the method (and some caveats) are discussed. Moreover, we present a series of evolutive snapshots, combining illustrative examples of both model and true planetary nebulae.

Conclusions. The zvpc radial-density reconstruction - added to tomography and 3D recovery developed at the Astronomical Observatory of Padua (Italy) - constitutes a very useful tool for looking more closely at the spatio-kinematics, physical conditions, ionic structure, and evolution of expanding nebulae.
\end{abstract}

Key words. planetary nebulae: general - ISM: kinematics and dynamics - ISM: structure

\section{Introduction}

The spatial distribution of gas constitutes the most important observational parameter for planetary nebulae (PNe): when combined with gas dynamics, it supplies fundamental information on the mechanisms and physical processes driving nebular evolution (mass-loss history, wind interaction, ionization, magnetic fields, binarity of the central star etc.) and allows us a direct comparison of each real object with theoretical evolutionary models, hydro-dynamical simulations, and photo-ionization codes.

In spite of this, the whole astronomical literature dedicated to $\mathrm{PNe}$ contains only a handful of papers dealing with their true spatial structure, which is generally obtained by deconvolving the volume emissivity from the apparent image using Abel's integration equation for spherically symmetric systems and ad hoc algorithms for inclined axi-symmetrical nebulae (Wilson \& Aller 1951; Lucy 1974; Soker et al. 1992; Volk \& Leahy 1993; Bremer 1995). These rare - quite rough and controversial - results stress the huge difficulties so far encountered in de-projecting the bi-dimensional appearance of a threedimensional structure (Aller 1994).

Recently, Sabbadin et al. (2005, and references therein) have suggested that the dynamical properties of the ionized gas represent the key for overcoming the stumbling block of de-projection

\footnotetext{
* Based on observations made with ESO Telescopes at the La Silla Observatories (program ID 65.I-0524), and TNG (Telescopio Nazionale Galileo) at La Palma, Canary Islands (program AOT10-10). We extensively apply the photo-ionization code CLOUDY, developed at the Institute of Astronomy of the Cambridge University (Ferland et al. 1998).

$\star \star$ Movies are available in electronic form at

http://www. edpsciences.org
}

in PNe and other types of expanding nebulae, like nova and supernova remnants, shells around Population I Wolf-Rayet stars, nebulae ejected by symbiotic stars, bubbles surrounding early spectral-type main sequence stars etc.

Historically, the general kinematical rule for the bright main shell of PNe goes back to Wilson (1950), based on Coudé spectra - no image-derotator - of 26 targets: the high-excitation zones expand more slowly than the low-excitation ones, and there is a direct correlation between the expansion velocity and the size of the monochromatic image.

Weedman (1968) secured Coudé spectra (+image-derotator) of a sub-set of Wilson's list (10 PNe), covered along the apparent major axis. Assuming a priori the prolate spheroid hypothesis with $a / b=1.5$, Weedman derived a typical expansion law of the type $V_{\exp }=s\left(R-R_{0}\right)$, where $s$ is the (positive) slope of the expansion velocity gradient and $R_{0}$ the radius at which $V_{\exp }=0$. In most cases, $R_{0} \simeq 0$ or $R_{0} \ll R_{\text {neb }}$ (i.e. the outflow is nearly ballistic, Hubble-type).

The classical papers by Wilson (1950) and Weedman (1968) belong to the heroic age of photographic plates. Later on, the introduction of linear 2D detectors at high efficiency (CCDs) greatly enhanced flux accuracy in a wide spectral range, and the use of echelle spectrographs allowed the observers to extend the analysis to large, faint objects. Even so, an odd dichotomy marks the whole of astronomical literature: high-resolution spectra of $\mathrm{PNe}$ are used to obtain either the detailed kinematics in a few ions (generally, [O III], H I, and [N II]) or the "average" nebular fluxes.

In the former case (i.e. kinematics), long-slit observations are performed through an interference filter to isolate a single order containing one or two nebular emissions. Valuable examples 
concern the kinematics of: 15 multiple shell PNe (Guerrero et al. 1998, single position angle (PA), instrumental spectral resolution $\Delta V=6.5 \mathrm{~km} \mathrm{~s}^{-1}$ ), NGC 2438 (Corradi et al. 2000, single PA, $\Delta V=4.3 \mathrm{~km} \mathrm{~s}^{-1}$ ), and MZ 3 (a symbiotic-star nebula), covered at various PA by Santander-Garcia et al. $\left(2004, \Delta V=6.0 \mathrm{~km} \mathrm{~s}^{-1}\right)$ and Guerrero et al. $\left(2004, \Delta V=8.0 \mathrm{~km} \mathrm{~s}^{-1}\right)$. We must notice, however, that the standard observational procedure of strongly reducing the useful spectral range by means of an interference filter represents an excessive precaution in most cases, since the PN is an emission-line object. As shown by Benetti et al. (2003) and Sabbadin et al. (2004), a PN larger (even much larger) than the order separation of the echellograms can be covered with a single exposure in the whole spectral range, the only limit (variable from one instrument to an other) being the effective superposition of spectral images belonging to different orders (and not the mere order separation).

In the latter dichotomous case (average fluxes from highdispersion spectroscopy), line intensities are integrated over the entire slit (whose length is smaller than order separation). They provide "mean" physical conditions and ionic and chemical abundances within the whole slice of nebula covered by the spectrograph, thus losing the detailed (i.e. pixel-to-pixel) information on kinematics and flux. Representative examples are given by Hyung \& Aller (1998) and Hyung et al. (2001).

A courageous effort to overcome the foregoing impasse has been performed by Gesicki \& Zijlstra (2000) and Gesicki et al. (2003, and references therein), who secured high-resolution spectra of a number of compact PNe (including Galactic bulge objects and nebulae in the Sagittarius galaxy and in the Magellanic Clouds) and combined integrated emission profiles of forbidden and recombination lines with the photo-ionization code of a spherical nebula (Torum model), inferring that acceleration is a very common property in the PNe innermost layers (i.e. "U"-shaped expansion profile), due to the dynamical contribution by the shocked, hot wind from the central star.

Unfortunately, a detailed comparative analysis (Sabbadin et al. 2005) proves that the "U"-shaped expansion profile is a spurious, incorrect result caused by a combination of unsuited assumptions, spatial resolution, and diagnostic choice.

All this confirms that, due to the nebular complexity and large stratification of the radiation and kinematics, the recovery of the spatio-kinematical structure needs observations:

(a) at adequate "relative" spatial (SS) and spectral (RR) resolutions ( $\mathrm{SS}=R / \Delta r, \mathrm{R}=$ apparent radius, $\Delta r=$ seeing + guiding; $\mathrm{RR}=V_{\exp } / \Delta V, \Delta V=$ instrumental spectral resolution);

(b) in a wide spectral range containing emissions at low, medium, and high ionization; and

(c) at several slit positions over the nebula,

to be combined with a straightforward and versatile method of analysis.

To this end, in 2000 we started a multi-PA spectroscopic survey of bright PNe in both hemispheres, observed with ESO NTT+EMMI (80 echelle orders covering the spectral range $\lambda \lambda 3900-8000 \AA$ with $\left.\Delta V=5.0 \mathrm{~km} \mathrm{~s}^{-1}\right)$ and Telescopio Nazionale Galileo (TNG)+SARG (54 orders, spectral range $\lambda \lambda 4600-8000 \AA, \Delta V=2.63 \mathrm{~km} \mathrm{~s}^{-1}$ ). The pixel-to-pixel analysis of flux and velocity in a large number of emissions gives the bidimensional ionic structure (tomography) of each nebular slice intercepted by the spectrograph slit; moreover, a 3D rendering program assembling all tomographic maps provides the accurate spatial distribution of (i) the kinematics; (ii) physical conditions ( $T_{\mathrm{e}}$ and $N_{\mathrm{e}}$ ); and (iii) the ionic and chemical abundances within the nebula.
Besides the - in fieri - detailed spatio-kinematical study of individual PNe (Ragazzoni et al. 2001; Turatto et al. 2002; Benetti et al. 2003; Sabbadin et al. 2004, 2005), we decided to investigate a general, long-lasting open problem: inferring the radial density profile in expanding nebulae.

This introductory, didactic paper contains a detailed procedure for determining the gas distribution in real PNe; it is structured as follows: Sect. 2 gives some basic theoretical notions, Sect. 3 describes the practical application to high-dispersion spectra, Sect. 4 presents a series of evolutionary snapshots for model- and true-PNe, Sect. 5 contains the general discussion, and Sect. 6 draws the conclusions.

In a future paper we will compare the density profiles observed in a representative sample of targets with the expectations coming from recent hydro-dynamical simulations and theoretical models, in order to disentangle the evolutive phenomenology and physical processes responsible for PNe shape and shaping.

\section{Theory}

Following Aller (1984), Pottasch (1984), and Osterbrock (1989), the absolute flux emitted in the line $\lambda$ by the elementary volume of a steady-state nebula is given by:

$F\left(\mathrm{X}^{m}, \lambda\right)=N_{\mathrm{e}} N\left(\mathrm{X}^{n}\right) f\left(\mathrm{X}^{m}, \lambda, N_{\mathrm{e}}, T_{\mathrm{e}}\right) \epsilon_{l}$,

where $T_{\mathrm{e}}$ is the electron temperature, $N_{\mathrm{e}}$ the electron density, $\mathrm{X}^{n}$ the ion involved in the transition, $f\left(\mathrm{X}^{m}, \lambda, N_{\mathrm{e}}, T_{\mathrm{e}}\right)$ the emissivity function for the line $\left(\mathrm{X}^{m}, \lambda\right)$, and $\epsilon_{l}$ the local filling factor (fractional volume actually filled by matter with electron density $N_{\mathrm{e}}$ ).

The emissivity function $f\left(\mathrm{X}^{m}, \lambda, N_{\mathrm{e}}, T_{\mathrm{e}}\right)$ depends on the excitation mechanism. Recombination through the capture of an electron (followed by a cascade to lower levels) and collisional excitation by an electron (followed by spontaneous radiation) are the main excitation processes in $\mathrm{PNe}^{1}$.

For recombination lines $(n=m+1$ in Eq. (1))

$f\left(\mathrm{X}^{m}, \lambda_{i j}, N_{\mathrm{e}}, T_{\mathrm{e}}\right)=\frac{h c}{\lambda_{i j}} \alpha_{\mathrm{eff}}\left(\lambda_{i j}\right)$,

where $\alpha_{\text {eff }}\left(\lambda_{i j}\right)$ is the effective line recombination coefficient (a weak function of $N_{\mathrm{e}}$ ) given by

$\alpha_{\text {eff }}\left(\lambda_{i j}\right)=B\left(\lambda_{i j}\right) \alpha_{\text {eff }}\left(\mathrm{X}_{i}^{m}\right)$

and $B\left(\lambda_{i j}\right)$, the branching ratio, by

$B\left(\lambda_{i j}\right)=\frac{A_{i j}}{\sum_{k<i} A_{i k}}$,

with $A_{i k}=$ probabilities for spontaneous radiative decay.

The emissivity function for collisionally excited lines (lowdensity case; $n=m$ in Eq. (1)) is

$f\left(\mathrm{X}^{m}, \lambda, T_{\mathrm{e}}\right)=\frac{h c}{\lambda} q_{\mathrm{coll}}(\lambda)$

1 We overlook other possible excitation mechanisms, namely, (a) Bowen resonance fluorescence (a photon emitted by one ion is absorbed by another and degraded in a cascade of emission lines via intermediate levels); (b) starlight and/or nebular continuum fluorescence (a strong local UV radiation field pumps an ion into an excited state); (c) dielectronic recombinations (a core electron is excited by capture of a free electron); and (d) charge-exchange reactions (an electron is exchanged during the collision of a ion with $\mathrm{H}$ or $\mathrm{He}$, the most abundant species). Their possible role in a specific application (i.e. abundance dichotomy from optical recombination and collisionally excited lines) will be introduced in Sect. 5 . 


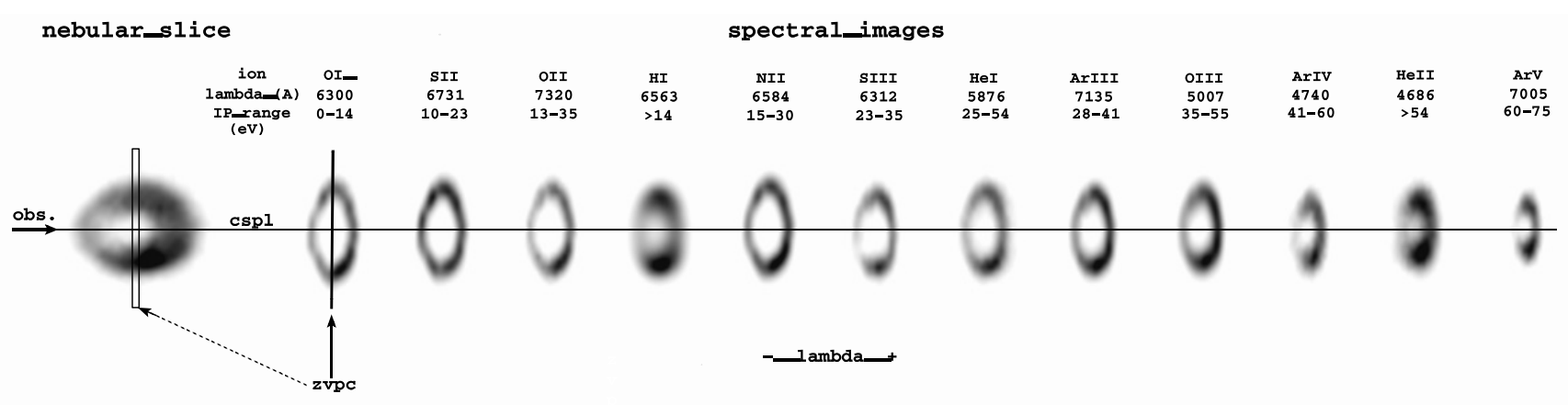

Fig. 1. Nebular slice intercepted by a spectrograph slit passing through the central star (left-most panel) and the related high-resolution spectral image (flux in arbitrary scale) in 12 ionic species arranged (left to right) in order of increasing ionization potential (IP; approximative range). The cspl is also indicated, as well as a zvpc (superimposed on the [O I] $\lambda 6300 \AA$ spectral image) with the corresponding part of real nebula (the rectangle centered on the nebular slice).

and $q_{\mathrm{coll}}(\lambda)$, the collisional excitation rate,

$q_{\text {coll }}(\lambda)=8.63 \times 10^{-6}\left(\frac{\Omega}{\omega_{1}}\right) T_{\mathrm{e}}^{-0.5} \mathrm{e}^{\frac{-\Delta E}{k T_{\mathrm{e}}}}$,

where $\Omega=$ collision strength of the transition averaged over the Maxwell distribution, $\omega_{1}=$ statistical weight of the lower level of the transition, $\Delta E=$ excitation potential of the upper level.

In general, $\alpha_{\text {eff }}(\lambda)$ has a weak, inverse dependence on $T_{\mathrm{e}}$, whereas $q_{\text {coll }}(\lambda)$, according to Eq. (6), is a strong, direct function of $T_{\mathrm{e}}$.

By relating the local electron density to an observable quantity (i.e. the local flux), Eq. (1) represents the starting point for the practical determination of the radial matter profile in realPNe (after de-projection of the apparent bi-dimensional morphology, as discussed in the next section).

\section{Practice}

Let us consider an optically thick, regularly expanding nebula at distance $D$, powered by a luminous post-AGB star at high temperature. A long slit centered on the exciting star intercepts the radial slice of nebula shown in the left-most panel of Fig. 1, whose high-resolution spectral image in different ions - also presented in Fig. 1 - enhances (a) the large stratification of the radiation and kinematics; and (b) the blurred appearance of $\mathrm{H} \mathrm{I}$, $\mathrm{He}$ I, and He II recombination lines, due to a mix of thermal motions, fine-structure and expansion velocity gradient across the nebula (more details are in Sabbadin et al. 2005; and Sect. 4.1) ${ }^{2}$. Moreover, Fig. 1 contains:

- the central-star-pixel-line (cspl), common to all PA, representing the matter projected at the apparent position of the central star, whose motion is purely radial;

- a zero-velocity-pixel-column (zvpc, superimposed on the [O I] spectral image at $\lambda 6300 \AA$ ), giving the spatial profile of the tangentially moving gas at the systemic radial velocity.

Hereafter we will focus on the zvpc. As clearly shown in the left-most panel of Fig. 1, it provides the ionic distribution within a sharp, central, well-defined portion of the radial slice of nebula selected by the spectrograph slit. Strictly speaking, the zvpc is marginally affected by the adjacent layers of the nebular slice and, at the same time, marginally contributes to the emission of

\footnotetext{
${ }^{2}$ The twelve spectral images in Fig. 1 refer to NGC 6741 at PA $=15^{\circ}$ (ESO NTT+EMMI).
}

the adjacent layers, since the instrumental spectral resolution is not infinite.

According to Eq. (1), the absolute flux emitted in line $\lambda$ by the nebular volume sampled by a single pixel of the zvpc is given by

$E\left(\mathrm{X}^{m}, \lambda\right)_{\mathrm{zvpc}}=F\left(\mathrm{X}^{m}, \lambda\right)_{\mathrm{zvpc}} \times V_{\mathrm{zvpc}}$,

where $V_{\mathrm{zvpc}}\left(\right.$ in $\left.\mathrm{cm}^{3}\right)$ is represented by the parallelepiped

$V_{\mathrm{zvpc}}=s \times w \times d$

with $s$, pixel size along the slit, given by

$s(\mathrm{~cm})=\operatorname{pixel}$ height $(\operatorname{arcsec}) \times \frac{D(\mathrm{~cm})}{206265}$,

$w$, pixel size perpendicular to the slit, by

$w(\mathrm{~cm})=\operatorname{slit} w i d t h(\operatorname{arcsec}) \times \frac{D(\mathrm{~cm})}{206265}$

and $d$, depth of the zvpc along the radial direction, in general by

$d(\mathrm{~cm})=\delta V\left(\mathrm{~km} \mathrm{~s}^{-1}\right) \times \frac{R(\lambda)(\operatorname{arcsec})}{V_{\exp }(\lambda)\left(\mathrm{km} \mathrm{s}^{-1}\right)} \times \frac{D(\mathrm{~cm})}{206265}$

and, for $V_{\exp }=A \times R^{\prime \prime}$, by

$d(\mathrm{~cm})=\frac{\delta V\left(\mathrm{~km} \mathrm{~s}^{-1}\right)}{A\left(\mathrm{~km} \mathrm{~s}^{-1} \operatorname{arcsec}^{-1}\right)} \times \frac{D(\mathrm{~cm})}{206265}$,

$\delta V\left(\mathrm{~km} \mathrm{~s}^{-1}\right)$ being the pixel spectral resolution.

The corresponding flux received by an observer is

$I\left(\mathrm{X}^{m}, \lambda\right)_{\mathrm{obs}}=\frac{I\left(\mathrm{X}^{m}, \lambda\right)_{\mathrm{corr}}}{10^{[c(\mathrm{H} \beta) \times f(\lambda)]}}$

with

$I\left(\mathrm{X}^{m}, \lambda\right)_{\mathrm{corr}}=\frac{E\left(\mathrm{X}^{m}, \lambda\right)_{\mathrm{zvpc}}}{4 \pi D(\mathrm{~cm})^{2}}$

where $c(\mathrm{H} \beta)$ is the logarithmic extinction at $\mathrm{H} \beta$ and $f(\lambda)$ the extinction coefficient given by Seaton (1979).

In the ionized nebula we have $N(\mathrm{H}) \simeq \mathrm{N}\left(\mathrm{H}^{+}\right)$and $N_{\mathrm{e}} \simeq$ $1.15 N\left(\mathrm{H}^{+}\right)$; thus, Eq. (7) can be written in the form ${ }^{3}$

$N_{\mathrm{e}}^{2} \epsilon_{\mathrm{l}} \propto\left[\frac{I\left(\mathrm{X}^{m}, \lambda\right)_{\text {corr }}}{f\left(\mathrm{X}^{m}, \lambda, N_{\mathrm{e}}, T_{\mathrm{e}}\right)}\right] \times \frac{N(\mathrm{H})}{N(\mathrm{X})} \times \operatorname{icf}\left(\mathrm{X}^{n}\right)$

${ }^{3}$ In this and the following equations, the proportionality constant is $\left[4 \pi(206265)^{3} \times A\left(\mathrm{~km} \mathrm{~s}^{-1} \operatorname{arcsec}^{-1}\right)\right] /\left[1.15 \times \delta V\left(\mathrm{~km} \mathrm{~s}^{-1}\right) \times\right.$ $s(\operatorname{arcsec}) \times w(\operatorname{arcsec}) \times D(\mathrm{~cm})]$ for $V_{\exp }=A \times R^{\prime \prime}$ and $\left[4 \pi(206265)^{3} \times V_{\exp }(\lambda)\left(\mathrm{km} \mathrm{s}^{-1}\right] /\left[1.15 \times \delta V\left(\mathrm{~km} \mathrm{~s}^{-1}\right) \times R(\lambda)(\operatorname{arcsec}) \times\right.\right.$ $s(\operatorname{arcsec}) \times w(\operatorname{arcsec}) \times D(\mathrm{~cm})]$ for $V_{\exp } \neq A \times R^{\prime \prime}$. 
where $\operatorname{icf}\left(\mathrm{X}^{n}\right)=\frac{N(\mathrm{X})}{N\left(\mathrm{X}^{n}\right)}$ is the ionization correcting factor.

Recalling that

$\sum_{i \leq z} \frac{1}{\mathrm{icf}\left(\mathrm{X}^{i}\right)}=1$

( $z=$ atomic number of the element $X)$, at last we obtain the general expression

$N_{\mathrm{e}}^{2} \epsilon_{1} \propto \frac{N(\mathrm{H})}{N(\mathrm{X})} \times \sum_{i \leq z} \frac{I\left(\mathrm{X}^{i}, \lambda\right)_{\mathrm{corr}}}{f\left(\mathrm{X}^{i}, \lambda, N_{\mathrm{e}}, T_{\mathrm{e}}\right)}$,

thereby providing $N_{\mathrm{e}} \epsilon_{l}^{1 / 2}$ in the zvpc from observable quantities (i.e. the pixel-to-pixel flux in one line for each ionic species of a given element).

In practice, for hydrogen, which has a single ionized state, Eq. (17) becomes

$N_{\mathrm{e}}^{2} \epsilon_{\mathrm{l}} \propto\left[\frac{I(\mathrm{HI}, 6563)_{\mathrm{corr}}}{\alpha_{\mathrm{eff}}(\mathrm{HI}, 6563)} \times \frac{6563}{h c}\right]$

or

$N_{\mathrm{e}}^{2} \epsilon_{\mathrm{l}} \propto\left[\frac{I(\mathrm{HI}, 4861)_{\text {corr }}}{\alpha_{\mathrm{eff}}(\mathrm{HI}, 4861)} \times \frac{4861}{h c}\right]$

or using any other H I Balmer recombination line.

For helium, the contribution of both ionization stages must be considered

$N_{\mathrm{e}}^{2} \epsilon_{1} \propto\left[C\left(\mathrm{He}^{+}\right)+C\left(\mathrm{He}^{++}\right)\right] \times \frac{N(\mathrm{H})}{N(\mathrm{He})}$

with

$C\left(\mathrm{He}^{+}\right)=\left[\frac{I(\mathrm{HeI}, 5876)_{\mathrm{corr}}}{\alpha_{\mathrm{eff}}(\mathrm{HeI}, 5876)} \times \frac{5876}{h c}\right]$

and

$C\left(\mathrm{He}^{++}\right)=\left[\frac{I(\mathrm{HeII}, 4686)_{\text {corr }}}{\alpha_{\mathrm{eff}}(\mathrm{HeII}, 4686)} \times \frac{4686}{h c}\right]$

or using any other pair of He I and He II recombination lines.

For heavier elements, we are forced to select collisionally excited emissions of suitable ionic sequences, due to the weakness of recombination lines, large stratification of the radiation, and incomplete ionic coverage of the echellograms. As tracers, we adopt $\mathrm{O}^{0}+\mathrm{O}^{+}+\mathrm{O}^{++}$for the external, low-to-medium ionization layers (IP range from 0 to $55 \mathrm{eV}$ ), and $\mathrm{Ar}^{++}+\mathrm{Ar}^{3+}+\mathrm{Ar}^{4+}$ for the internal, medium-to-high ionization ones (IP range from 28 to $75 \mathrm{eV})^{4}$.

From oxygen

$N_{\mathrm{e}}^{2} \epsilon_{1} \propto\left[C\left(\mathrm{O}^{0}\right)+C\left(\mathrm{O}^{+}\right)+C\left(\mathrm{O}^{++}\right)\right] \times \frac{N(\mathrm{H})}{N(\mathrm{O})}$

with

$C\left(\mathrm{O}^{0}\right)=\left[\frac{I(\mathrm{OI}, 6300)_{\mathrm{corr}}}{q_{\mathrm{coll}}(\mathrm{OI}, 6300)} \times \frac{6300}{h c}\right]$,

${ }^{4}$ Note, however, that ionic species higher than $\mathrm{Ar}^{4+}$ are expected in the innermost layers of a PN powered by a central star with $T_{*} \geq$ $100000 \mathrm{~K}$. In this case, $\lambda 7005 \AA$ and/or $\lambda 6435 \AA$ of $\mathrm{Ar}^{4+}$ become poor density diagnostics for the internal, highest-excitation regions ( $\lambda 3425 \AA$ of [Ne V], IP range 97 to $126 \mathrm{eV}$, should be preferable).
$C\left(\mathrm{O}^{+}\right)=\left[\frac{I(\mathrm{OII}, 7320)_{\mathrm{corr}}}{q_{\text {coll }}(\mathrm{OII}, 7320)} \times \frac{7320}{h c}\right]$

(7320 corresponding to $\lambda 7320.121 \AA$, the strongest component of the [O II] red quartet) and

$C\left(\mathrm{O}^{++}\right)=\left[\frac{I(\mathrm{OIII}, 5007)_{\mathrm{corr}}}{q_{\mathrm{coll}}(\mathrm{OIII}, 5007)} \times \frac{5007}{h c}\right]$

From argon

$N_{\mathrm{e}}^{2} \epsilon_{1} \propto\left[C\left(\mathrm{Ar}^{++}\right)+C\left(\mathrm{Ar}^{3+}\right)+C\left(\mathrm{Ar}^{4+}\right)\right] \times \frac{N(\mathrm{H})}{N(\mathrm{Ar})}$

with

$C\left(\mathrm{Ar}^{++}\right)=\left[\frac{I(\text { ArIII, 7135) }}{q_{\text {coll }}(\text { ArIII }, 7135)} \times \frac{7135}{h c}\right]$

$C\left(\mathrm{Ar}^{3+}\right)=\left[\frac{I(\mathrm{ArIV}, 4740)_{\mathrm{corr}}}{q_{\mathrm{coll}}(\mathrm{ArIV}, 4740)} \times \frac{4740}{h c}\right]$

and

$C\left(\mathrm{Ar}^{4+}\right)=\left[\frac{I(\mathrm{ArV}, 7005)_{\mathrm{corr}}}{q_{\mathrm{coll}}(\mathrm{ArV}, 7005)} \times \frac{7005}{h c}\right]$.

Besides Eqs. (17) to (30), the $N_{\mathrm{e}}$ profile in the zvpc is also given by line-intensity ratios of ions in $\mathrm{p}^{3}$ configuration, like $\lambda 6717 \AA / \lambda 6731 \AA$ of [S II] for low-ionization regions and $\lambda 4711 \AA / \lambda 4740 \AA$ of [Ar IV] for the high-ionization ones. However, these diagnostics are generally weak in PNe: $N_{\mathrm{e}}[\mathrm{S}$ II] and $N_{\mathrm{e}}$ [Ar IV] can be derived only at (or close to) the corresponding intensity peak.

Summing up, in the most favorable case (i.e. long-slit, wide spectral range, absolute flux calibrated echellograms of a $\mathrm{PN}$ at known distance), we infer both $N_{\mathrm{e}}(\mathrm{zvpc})$ and $\epsilon_{l}(\mathrm{zvpc})$ by combining Eqs. (17) to (30) with $N_{\mathrm{e}}\left[\mathrm{S}\right.$ II] and $N_{\mathrm{e}}$ [Ar IV]. In this case, (a) chemical abundances come from line fluxes integrated over the whole spatial and spectral profile, Alexander \& Balick (1997), Perinotto et al. (1998); and (b) the $T_{\mathrm{e}}$ (zvpc) distribution comes from diagnostics of ions in $\mathrm{p}^{2}$ and $\mathrm{p}^{4}$ configurations, like $\lambda 6584 \AA / \lambda 5755 \AA$ of [N II] for low-ionization regions and $\lambda 5007 \AA / \lambda 4363 \AA$ of [O III] for the high-ionization ones, Turatto et al. 2002; Benetti et al. 2003). On the other hand, in the most unfavorable case (i.e. flux un-calibrated spectra of a PN at an unknown distance) Eqs. (17) to (30) provide the "relative" $N_{\mathrm{e}} \epsilon_{l}^{1 / 2}$ profile in the $\mathrm{zvpc}^{5}$.

5 Moreover, (a) the combination of Eqs. (15) and (17) gives the radial ionization structure of each element (more details are in Sect. 4.1) and (b) the accurate $\mathrm{He} / \mathrm{O}$ and $\mathrm{Ar} / \mathrm{O}$ radial chemical profiles can be obtained by assembling Eqs. (18)-(20), (23) and (27); we will go more deeply into this topic in a future, dedicated paper. 
In order

- to check the general validity and applicability of our $N_{\mathrm{e}}$ reconstruction method for different physical and evolutionary conditions of the ionized gas; and

- to tackle the various "practical" problems connected with the observations of a true-PN (e.g. finite spatial and spectral resolutions and incomplete ionic coverage of the echellograms, thermal motions, turbulence and fine-structure of recombination lines),

in the next section we

a) will expose an arbitrary and imaginary model nebula to a series of UV stellar fluxes; and

b) will analyse the zvpc-flux profile in a few representative true-PNe.

\section{Theory vs. practice}

According to the most recent theoretical evolutionary models and radiation-hydrodynamics simulations, a PN is the result of the synergistic effects of ionization and fast wind on the gas ejected during the superwind phase of an AGB-star (Blöcker \& Schönberner 1990; Vassiliadis \& Wood 1994; Blöcker 1995; Marigo et al. 2001; Perinotto et al. 2004b).

By combining the large variation of post-AGB stellar characteristics (temperature and luminosity) with the gradual nebular dilution due to expansion, we can sketch the evolution of a "normal" PN as follows. In the early phases the nebula is optically thick to the UV stellar radiation, then the gas gradually becomes optically thin until the quick luminosity decline of the hot star at the end of the hydrogen-shell nuclear burning, leading to a recombination of the external nebular layers. Later on, the PN enters in a final re-ionization phase, because of the slowing down in the stellar luminosity decline.

The recombination and re-ionization phases are absent in a low-mass PN ionized by a low-mass, slowly evolving central star, whereas massive PNe powered by a high-mass, fast evolving post-AGB star never become optically thin to the UV stellar radiation. A further complication is represented by a possible PN "rejuvenation": the hydrogen-deficient post-AGB star can suffer a late thermal pulse during the motion towards the white-dwarf region, mixing and burning hydrogen on a convective turn-over time scale and producing a "born-again" PN (Blöcker 1995, 2001; Herwig et al. 1999).

Of course, all this represents an over-simplification, as each real $\mathrm{PN}$ is an extremely complex and inhomogeneous structure. It can be optically thick at some radial directions (e.g. along and close to the dense equatorial regions) and thin at other directions (e.g. along the low-density polar caps).

For illustrative purposes, we expose a single, "standard" radial matter profile (representing the gas distribution of an ideal, average, spherically symmetric nebula) to a series of UV stellar fluxes mimicing the evolution of a post-AGB star. The resulting ionization structure of each "model-PN" snapshot, provided by the photo-ionization code CLOUDY (Ferland et al. 1998), will be analysed in detail and coupled with the zvpc-flux profiles observed in a true-PN at a comparable evolutionary phase. The input parameters for the model nebula are summarized in Table 1, whereas Table 2 contains the integrated flux in the main optical emissions (relative to $I(\mathrm{H} \beta)=100$ ), the absolute $\mathrm{H} \beta$ flux, and the resulting ionized mass at four representative evolutionary steps.

Let us start with a "quite young" PN close to the thick-thin transition, ionized by a luminous post-AGB star of $0.605 M_{\odot}$
Table 1. Input parameters for the adopted, spherically symmetric model nebula (CLOUDY)

\begin{tabular}{ll}
\hline \hline Gas density profile & $\begin{array}{l}\text { The same, standard and arbitrary, } \\
\text { double-peak+halo distribution at } \\
\text { all four evolutionary steps } \\
\text { (see Figs. 2, 4-6; cf. Sect. 4) }\end{array}$ \\
Chemical abundances & $\mathrm{He}=-0.958, \mathrm{C}=-3.30, \mathrm{~N}=-3.75$, \\
$\log [N(\mathrm{X}) / N(\mathrm{H})]$ & $\mathrm{O}=-3.30, \mathrm{~F}=-6.522, \mathrm{Ne}=-3.90$, \\
& $\mathrm{Na}=-5.52, \mathrm{Mg}=-5.30, \mathrm{Al}=-6.568$, \\
& $\mathrm{Si}=-5.00, \mathrm{P}=-6.70, \mathrm{~S}=-5.10$, \\
& $\mathrm{Cl}=-6.66, \mathrm{Ar}=-5.60, \mathrm{~K}=-7.11$, \\
& $\mathrm{Ca}=-6.64, \mathrm{Fe}=-6.30$, \\
& other elements $=\mathrm{CLOUDY}$ default \\
& $\mathrm{CLOUDY}$ default \\
Dust & 1.0 \\
Local filling factor &
\end{tabular}

at a moderate temperature $\left(T_{*}=50000 \mathrm{~K}\right)$, thus postponing to Sect. 4.4 the special case combining proto-PNe and lowexcitation "born again" $\mathrm{PNe}$.

\subsection{Early PN evolution}

In this first evolutionary snapshot, the ionizing engine of the adopted, spherically symmetric matter distribution (Table 1) is a hydrogen-burning post-AGB star of $0.605 M_{\odot}$ with $T_{*}=$ $50000 \mathrm{~K}, L_{*} / L_{\odot}=6500$, and $t_{\mathrm{SW}}=$ time elapsed from the end of the superwind $\simeq 3000 \mathrm{yr}$ (Blöcker 1995, and references therein).

The results for the model nebula (summarized in Table 2, Col. 4) are graphically shown in Fig. 2, where:

- top panel: standard radial matter profile and resulting trend for the physical conditions;

- next-to-top panel: radial ionization structure of the gas;

- next-to-bottom panel: absolute flux in the main emissions;

- bottom panel: $N_{\mathrm{e}} \epsilon_{l}^{1 / 2}$ distribution given by Eqs. (17) to (30).

In our case $N_{\mathrm{e}} \epsilon_{l}^{1 / 2}=N_{\mathrm{e}}$, since we have assumed $\epsilon_{l}=1$ (see Table 1).

High ionization emissions, like He II and Ar V, are very weak or absent in Fig. 2, as expected from the "moderate" star temperature, whereas the enhancement of [O I] and [O II] at the extreme nebular edge indicates that the model-PN is nearly optically thin to the UV stellar radiation.

The value of $T_{\mathrm{e}}$ is high ( $>10000 \mathrm{~K}$ ) in the innermost, tenuous layers, then rapidly decreases to $\simeq 8000 \mathrm{~K}$ in the densest regions, slightly increases further $(\simeq 9500 \mathrm{~K})$, and definitively falls in the outermost, partially neutral strata.

The bottom panel of Fig. 2 shows the $N_{\mathrm{e}} \epsilon_{l}^{1 / 2}$ profile obtained from $\mathrm{H}^{+}, \mathrm{He}^{+}+\mathrm{He}^{++}, \mathrm{O}^{0}+\mathrm{O}^{+}+\mathrm{O}^{++}$, and $\mathrm{Ar}^{++}+\mathrm{Ar}^{3+}+\mathrm{Ar}^{4+}$ (through Eqs. (17) to (30) and assuming $T_{\mathrm{e}}=8000 \mathrm{~K}$ for all ions, except in the innermost nebular regions, where $T_{\mathrm{e}}\left(\mathrm{He}^{++}\right)=$ $\left.T_{\mathrm{e}}\left(\mathrm{Ar}^{4+}\right)=13000 \mathrm{~K}\right)$. Please note the satisfactory agreement with the input radial-density distribution (top panel of Fig. 2), confirming the general validity (and applicability) of the procedure developed in Sect. $3^{6}$.

6 Although the adopted matter distribution of the model nebula is open to criticism (it is arbitrary, schematic, static, etc.), we underline that the choice of more sophisticated and/or time-dependent density profiles does not modify the general results illustrated here and in the following sub-sections. 
Table 2. Four-step model-PN evolution. Integrated intensity of representative nebular emissions (relative to $I(\mathrm{H} \beta)=100)$, absolute $\mathrm{H} \beta$ flux, and ionized mass for the adopted, spherically symmetric nebula (Table 1 ) powered by a hydrogen-burning post-AGB star of $0.605 M_{\odot}$.

\begin{tabular}{|c|c|c|c|c|c|}
\hline \multirow[t]{2}{*}{$\lambda(\AA)$} & \multirow[t]{2}{*}{ Ion } & \multicolumn{3}{|c|}{$I(\lambda)$} & \\
\hline & & $\begin{array}{c}\text { Sect. } 4.4 \\
\text { proto-PN + } \\
\text { "born-again" low-exc.-PN } \\
\text { (very thick) } \\
T_{*}=25000 \mathrm{~K} \\
L_{*} / L_{\odot}=6500\end{array}$ & $\begin{array}{c}\text { Sect. } 4.1 \\
\text { early-PN } \\
\text { (nearly thin) } \\
T_{*}=50000 \mathrm{~K} \\
L_{*} / L_{\odot}=6500\end{array}$ & $\begin{array}{c}\text { Sect. } 4.2 \\
\text { intermediate-PN } \\
\text { (thin) } \\
T_{*}=100000 \mathrm{~K} \\
L_{*} / L_{\odot}=6500\end{array}$ & $\begin{array}{c}\text { Sect. } 4.3 \\
\text { late-PN } \\
\text { (recombining) } \\
T_{*}=150000 \mathrm{~K} \\
L_{*} / L_{\odot}=1000\end{array}$ \\
\hline 3425 & {$[\mathrm{Ne} \mathrm{V}]$} & 0.0 & 0.0 & 4.1 & 14.7 \\
\hline $3726+29$ & {$[\mathrm{O} \mathrm{II}]$} & 133.3 & 231.2 & 73.8 & 587.5 \\
\hline 3968 & [Ne III] & 0.02 & 15.2 & 49.8 & 64.9 \\
\hline 4686 & $\mathrm{He}$ II & 0.0 & 0.25 & 19.0 & 42.2 \\
\hline 4740 & [Ar IV] & 0.0 & 0.22 & 3.4 & 2.9 \\
\hline 4861 & H I & 100.0 & 100.0 & 100.0 & 100.0 \\
\hline 5007 & [O III] & 5.5 & 490.0 & 1510.0 & 1312.2 \\
\hline 5876 & $\mathrm{He} \mathrm{I}$ & 3.11 & 15.5 & 12.6 & 10.5 \\
\hline 6300 & {$[\mathrm{O} I]$} & 1.3 & 2.5 & 0.1 & 26.3 \\
\hline 6563 & $\mathrm{H} \mathrm{I}$ & 297.2 & 291.7 & 285.7 & 288.4 \\
\hline 6584 & {$[\mathrm{~N}$ II $]$} & 265.5 & 169.0 & 37.3 & 434.5 \\
\hline $6717+31$ & {$[\mathrm{~S} \mathrm{II}]$} & 43.1 & 24.4 & 12.4 & 107.3 \\
\hline 7005 & [Ar V] & 0.0 & 0.0 & 0.77 & 1.05 \\
\hline 7135 & [Ar III] & 1.6 & 17.4 & 21.0 & 30.7 \\
\hline 7320 & {$[\mathrm{O} \mathrm{II}]$} & 0.8 & 2.2 & 1.0 & 9.8 \\
\hline $\log E(\mathrm{H} \beta)$ & $\left(\mathrm{erg} \mathrm{s}^{-1}\right)$ & 34.549 & 35.057 & 35.019 & 34.321 \\
\hline$M_{\text {ion }}$ & $\left(M_{\odot}\right)$ & 0.10 & 0.38 & 0.40 & $\begin{array}{l}0.07^{*} \\
\text { * excluding the ionized mass } \\
\text { of the recombining halo }\end{array}$ \\
\hline
\end{tabular}

From theory to practice: NGC 6572 (PNG 034.6+11.8, Acker et al. 1992) is a representative example of PN in quite an early evolutionary phase. It consists of a bright $(\log F(\mathrm{H} \beta=$ $-9.82 \mathrm{erg} \mathrm{cm}^{-2} \mathrm{~s}^{-1}$ ), moderate excitation (excitation class 5, Hyung et al. 1994), high-density ( $\log N_{\mathrm{e}}=3.7$ to 4.4 , Hyung et al. 1994; Liu et al. 2004), irregular disk $\left(d \simeq 5.0^{\prime \prime}\right)$ embedded in a faint envelope $\left(10.0^{\prime \prime} \times 16.0^{\prime \prime}\right)$ elongated in $\mathrm{PA} \simeq 0^{\circ}$.

Wilson (1950) measured $V_{\exp }([\mathrm{O} \mathrm{I}])=16.0 \mathrm{~km} \mathrm{~s}^{-1}$, $V_{\exp }([\mathrm{O}$ II $])=16.85 \mathrm{~km} \mathrm{~s}^{-1}, V_{\exp }([\mathrm{S} \mathrm{II}])=15.5 \mathrm{~km} \mathrm{~s}^{-1}$, and $V_{\exp }([\mathrm{N} \mathrm{II}])=14.75 \mathrm{~km} \mathrm{~s}^{-1}$; Weedman (1968) gave the general expansion law $V_{\text {exp }}\left(\mathrm{km} \mathrm{s}^{-1}\right)=3.4 \times R^{\prime \prime}$; Miranda et al. (1999, $\mathrm{H} \alpha+[\mathrm{N} \mathrm{II}]$ echellograms at $3 \mathrm{PA} ; \Delta V=8.0 \mathrm{~km} \mathrm{~s}^{-1}$ ) proved the presence of a collimated bipolar outflow along and close to the apparent major axis and of an equatorial density enhancement of toroidal structure with $V_{\exp }(\mathrm{H} \alpha)=14.0 \mathrm{~km} \mathrm{~s}^{-1}$ and $V_{\exp }([\mathrm{N} \mathrm{II}])=18.0 \mathrm{~km} \mathrm{~s}^{-1}$.

The exciting star of NGC 6572 has $m_{B} \simeq 13.0$, a spectral type Of/WR (Acker et al. 1992), $\log \left(T_{Z} \mathrm{H} \mathrm{I}\right) \simeq \log \left(T_{Z} \mathrm{He}\right.$ II $) \simeq$ 4.83 (Phillips 2003, and references therein), and $\log L_{*} / L_{\odot}=$ $3.50( \pm 0.20)$ (for an assumed distance of $1200 \mathrm{pc}$, Hajian et al. 1995; Kawamura \& Masson 1996; Miranda et al. 1999; and $c(\mathrm{H} \beta) \simeq 0.45$, Hyung et al. 1994; Liu et al. 2004). It is losing mass at a high, although uncertain, rate $\left(1.0 \times 10^{-9} M_{\odot} \mathrm{yr}^{-1}\right.$, $6.3 \times 10^{-9} M_{\odot} \mathrm{yr}^{-1}$, and $3.0 \times 10^{-8} M_{\odot} \mathrm{yr}^{-1}$, according to Cerruti-Sola \& Perinotto 1985; Hutsemekers \& Surdej 1989; and Modigliani et al. 1993, respectively), and a terminal wind velocity of $1800 \mathrm{~km} \mathrm{~s}^{-1}$.

The closeness of $\mathrm{H}$ I and He II Zanstra temperature of the star and the enhancement of low ionization species ([O I], [O II] etc.) at the nebular edge suggest that NGC 6572 is still optically thick to the UV stellar radiation.

We observed the nebula at six equally spaced PA with the high-resolution cross dispersed echelle spectrograph SARG
(Gratton et al. 2001) mounted at TNG (Telescopio Nazionale Galileo), under non-photometric sky conditions and a seeing ranging between $0.50^{\prime \prime}$ and $0.70^{\prime \prime}$. The spectrograph slit $\left(0.40^{\prime \prime}\right.$ wide and $26.7^{\prime \prime}$ long) was centered on the exciting star. The echellograms (exposure time $360 \mathrm{~s}$ ) covered the spectral range $\lambda \lambda 4600-8000 \AA$ with instrumental resolution $\Delta V \simeq$ $114000\left(2.63 \mathrm{~km} \mathrm{~s}^{-1}\right)$, pixel spectral scale $\delta V=1.20 \mathrm{~km} \mathrm{~s}^{-1}$, and pixel spatial scale $s=0.167$ arcsec. The spectra were reprocessed according to the straightforward procedure described by Turatto et al. (2002), including bias, zero-order flat field and distortion corrections, and wavelength and relative flux ${ }^{7}$ calibrations.

Let us select the spectrum at $\mathrm{PA}=102^{\circ}$, i.e. close to the apparent minor axis of NGC 6572, since, according to the de-projection criteria introduced by Sabbadin et al. (2005), $R_{\text {zvpc }}$ (apparent minor axis) $\simeq R_{\text {cspl }}$. The cspl vs. zvpc relation for NGC 6572 at $P A=102^{\circ}$ - based on ten ionic species, i.e. $\mathrm{H}^{+}, \mathrm{He}^{+}, \mathrm{O}^{0}, \mathrm{O}^{+}, \mathrm{O}^{++}, \mathrm{N}^{+}, \mathrm{S}^{+}, \mathrm{S}^{++}, \mathrm{Ar}^{++}$, and $\mathrm{Ar}^{+3}-$ gives the expansion law $V_{\text {exp }}\left(\mathrm{km} \mathrm{s}^{-1}\right)=8.0( \pm 1) \times R^{\prime \prime}$, whose slope is much larger than Weedman's (1968) report ${ }^{8}$. This implies that the dynamical age of the nebula is about $750 \mathrm{yr}$ and the true age $1200-1400$ yr.

Due to the nebular compactness and low expansion velocity $(\mathrm{SS} \simeq \mathrm{RR} \simeq 5$, see Sect. 1$)$, the spectral images must be carefully de-blurred. To this end we apply the Richardson-Lucy

\footnotetext{
7 Only "relative" fluxes are considered here, since the observations have been secured in non-photometric sky conditions. Note, however, that an absolute calibration can be obtained by means of the $\mathrm{H} \beta, \mathrm{H} \alpha$, and [O III] HST imagery of NGC 6572; in fact, the absolute flux within the $0.40^{\prime \prime} \times 26.7^{\prime \prime}$ rectangle of HST image intercepted by the spectrograph slit matches the integrated flux of the corresponding spectral emission.

8 Weedman obtained $V_{\exp }\left(\mathrm{km} \mathrm{s}^{-1}\right)=3.4 \times R^{\prime \prime}$ assuming $R_{\text {zvpc }}$ (apparent major axis) $\simeq R_{\text {cspl }}$.
} 


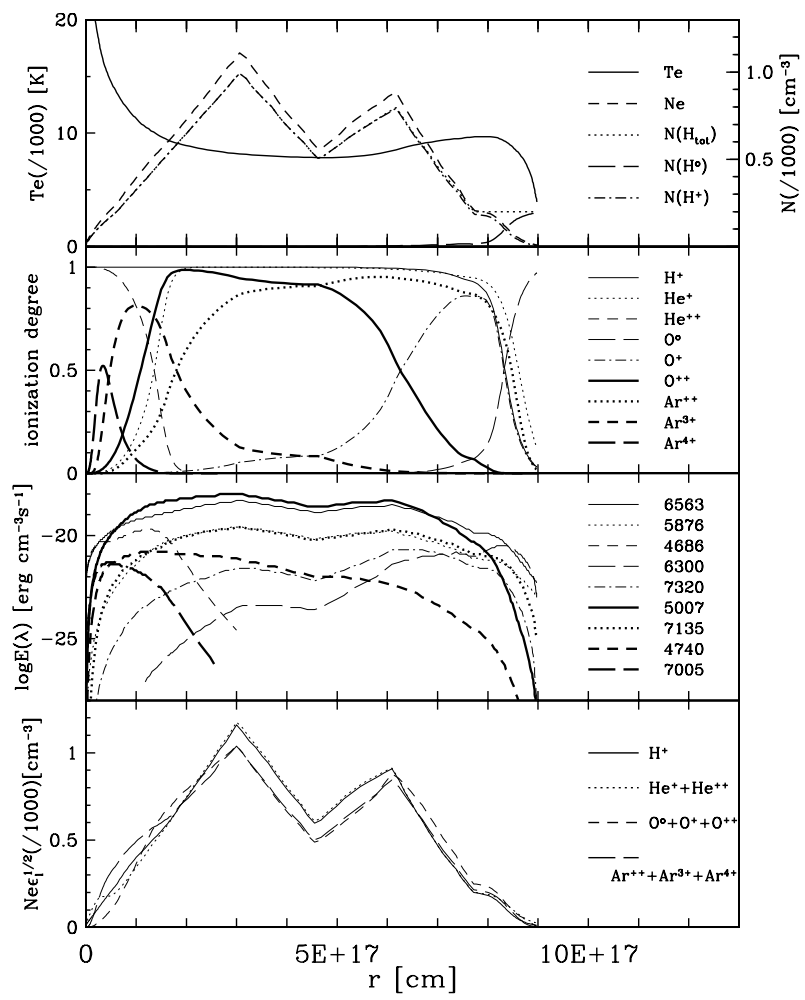

Fig. 2. Early model-PN evolution (Col. 4 of Table 2). General properties of the standard, spherically symmetric model nebula (Table 1) powered by a central star with $T_{*}=50000 \mathrm{~K}$ and $L_{*} / L_{\odot}=6500$. Top panel: adopted radial matter profile and resulting trend for the physical conditions. Next-to-top panel: radial ionization structure of the gas. Next-to-bottom panel: absolute flux in the main emissions. Bottom panel: $N_{\mathrm{e}} \epsilon_{l}^{1 / 2}$ distribution given by Eqs. (17) to (30), assuming $T_{\mathrm{e}}=8000 \mathrm{~K}$ for all ions, except in the innermost nebular regions, where $T_{\mathrm{e}}\left(\mathrm{He}^{++}\right)=T_{\mathrm{e}}\left(\mathrm{Ar}^{4+}\right)=13000 \mathrm{~K} . N_{\mathrm{e}} \epsilon_{l}^{1 / 2}$ can be directly compared with the electron density profile shown in the top panel, since we have adopted $\epsilon_{l}$ (model nebula) $=1$.

algorithm (Richardson 1972; Lucy 1974) with the point-spread function given by a bi-dimensional Gaussian profile characterized by $W_{\text {seeing }}$ and $W(\mathrm{vel})_{\text {tot }}$, where:

$W_{\text {seeing }}-F W H M$ of the instrumental profile along the spatial axis of the echellograms - corresponds to $0.52^{\prime \prime}$ (3.11 pixel),

$W(\mathrm{vel})_{\text {tot }}-F W H M$ of the broadening function along the velocity axis of the echellograms - is given by

$W(\text { vel })_{\text {tot }}=\left[W_{\text {SARG }}^{2}+W_{\text {thermal }}^{2}+W_{\text {turb }}^{2}+W_{\text {fine-s. }}^{2}\right]^{1 / 2}$

with

$W_{\text {SARG }}=$ instrumental resolution $=\Delta V=2.63 \mathrm{~km} \mathrm{~s}^{-1}$;

$W_{\text {thermal }}=$ thermal motions $=21.6 \times 10^{-2} \times T_{\mathrm{e}}^{0.5} \times m^{-0.5} \mathrm{~km} \mathrm{~s}^{-1}$

( $m=$ atomic weight of the element); we assume $T_{\mathrm{e}}=$ $11000 \mathrm{~K}$;

$W_{\text {turb }}=$ random, small-scale motions $=5.0 \mathrm{~km} \mathrm{~s}^{-1}$ (adopted value);

$W_{\text {fine-s. }}=$ fine-structure of recombination lines $=7.7 \mathrm{~km} \mathrm{~s}^{-1}$ for $\mathrm{H} \beta$ and $\mathrm{H} \alpha$ and $5.0 \mathrm{~km} \mathrm{~s}^{-1}$ for $\lambda 4686 \AA$ of He II (after suppression of the $20 \mathrm{~km} \mathrm{~s}^{-1}$ blue-shifted tail; for details, see Sabbadin et al. 2005) and $25876 \AA$ of He I.

$W(\text { vel })_{\text {tot }}$ amounts to $\simeq 23 \mathrm{~km} \mathrm{~s}^{-1}(\simeq 19$ pixel $)$ for $\mathrm{H} \beta$ and $\mathrm{H} \alpha$, $\simeq 13 \mathrm{~km} \mathrm{~s}^{-1}$ ( $\simeq 11$ pixel) for $\lambda 5876 \AA$ of He I and $\lambda 4686 \AA$ of

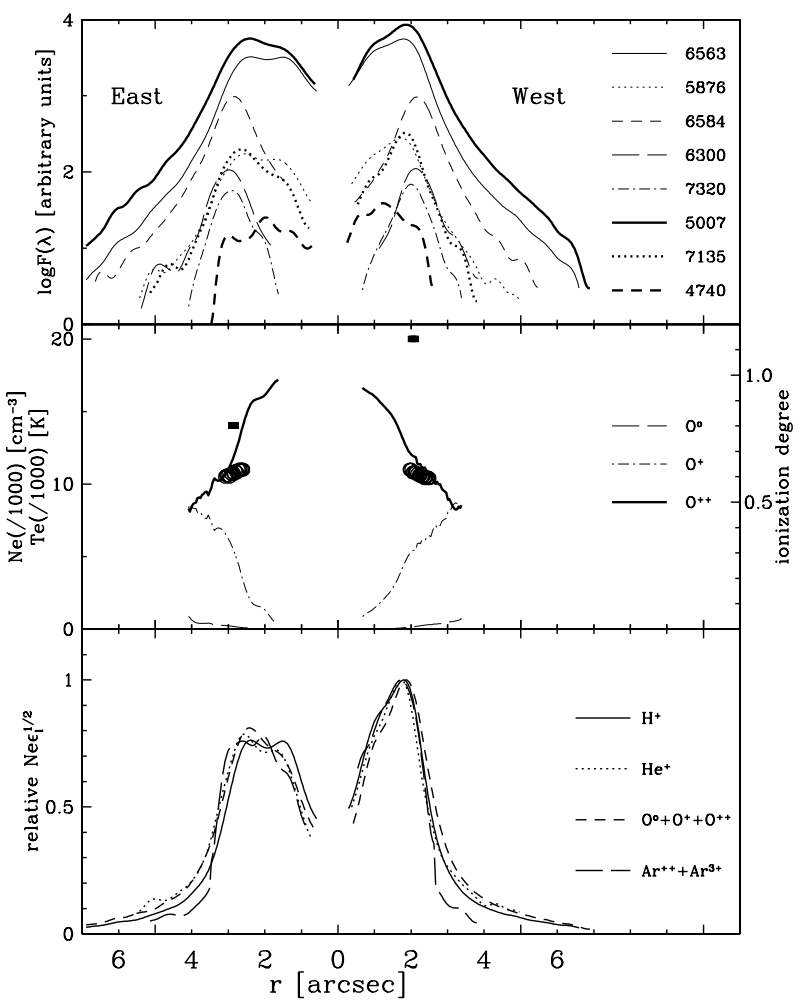

Fig. 3. Early evolution of a true-PN. General properties in the zvpc of NGC 6572 at PA $=102^{\circ}$ (apparent minor axis). Top panel: flux distribution of the main nebular emissions across the whole nebula (in arbitrary scale and corrected for $c(\mathrm{H} \beta)=0.45)$. Middle panel: $T_{\mathrm{e}}([\mathrm{N} \mathrm{II}])$ (circles) and $N_{\mathrm{e}}$ ([S II]) (squares) at the corresponding line peak and ionization structure of oxygen (from Eq. (32) and assuming $T_{\mathrm{e}}=11000 \mathrm{~K}$ across the whole nebula). Bottom panel: relative $N_{\mathrm{e}} \epsilon_{l}^{1 / 2}$ profile from $\lambda 6563 \AA$ $\left(\mathrm{H}^{+}\right), \lambda 5876 \AA\left(\mathrm{He}^{+}\right), \lambda 6300 \AA\left(\mathrm{O}^{0}\right)+\lambda 7320 \AA\left(\mathrm{O}^{+}\right)+\lambda 5007 \AA\left(\mathrm{O}^{++}\right)$, and $\lambda 7135 \AA\left(\mathrm{Ar}^{++}\right)+\lambda 4740 \AA\left(\mathrm{Ar}^{3+}\right)$ (through Eqs. (17) to (30)). The zvpc crosses the entire true-PN and provides the gas characteristics in two opposite radial directions in the plane of the sky. The central star position is at $r=0.0 \operatorname{arcsec}$ and the slit orientation is shown in the top panel.

He II, and $\simeq 7$ to $8 \mathrm{~km} \mathrm{~s}^{-1}$ ( 6 to 7 pixel) for the forbidden lines of heavier elements $(\mathrm{N}, \mathrm{O}, \mathrm{S}, \mathrm{Ar}$, etc.). The detailed results for the zvpc of NGC 6572 at PA $=102^{\circ}$ are illustrated in Fig. 3, whose top panel contains the flux distribution across the whole nebula ${ }^{9}$ (in arbitrary scale and corrected for $c(\mathrm{H} \beta)=0.45$ ) of the main nebular emissions. Note the absence of the highest ionization lines: $\lambda 6435 \AA$ and $\lambda 7005 \AA$ of [Ar V] and $\lambda 6560 \AA$ of He II are below the detection limit, whereas $\lambda 4686 \AA$ of He II lies at the extreme blue edge of the frame and is too noisy.

The middle panel of Fig. 3 shows:

(a) $T_{\mathrm{e}}([\mathrm{N} \mathrm{II}])$ (circles) and $N_{\mathrm{e}}([\mathrm{S} \mathrm{II}])$ (squares) at the corresponding line peak. Both diagnostics are uncertain, since the [N II] auroral line is quite faint and the $\lambda 6717 \AA / \lambda 6731 \AA$ intensity ratio is close to the high-density limit, where collisional

9 According to Fig. 1, the zvpc of a true-PN crosses the entire object and provides two independent density profiles in opposite directions (in Fig. 3 the central star position is at $r=0.0$ arcsec and the slit orientation is indicated in the top panel). Of course, the two zvpc gas distributions should coincide for a spherically symmetric nebula (as in the case of the standard model-PN adopted in this paper; that is why Fig. 2 and the next figures concerning the model-PN show the radial density profile in a single direction). 
de-excitations dominate and the diagnostic is a weak function of $N_{\mathrm{e}}$;

(b) the radial ionization structure of oxygen. According to Eqs. (15) and (17), it is given by

$$
\frac{N\left(\mathrm{X}^{i}\right)}{N(\mathrm{X})}=\frac{1}{\operatorname{icf}\left(\mathrm{X}^{i}\right)}=\frac{\frac{I\left(\mathrm{X}^{i}, \lambda\right)_{\text {corr }}}{f\left(\mathrm{X}^{i}, \lambda, N_{\mathrm{e}}, T_{\mathrm{e}}\right)}}{\sum_{\mathrm{i}} \frac{I\left(\mathrm{X}^{i}, \lambda\right)_{\text {corr }}}{f\left(\mathrm{X}^{i}, \lambda, N_{\mathrm{e}}, T_{\mathrm{e}}\right)}}
$$

with $i=0,1$, and 2 (moreover, we take $T_{\mathrm{e}}=11000 \mathrm{~K}$ in the whole nebula). Although Eq. (32) is stricty valid in the portion of zvpc containing the emission of all the three ionic species, we can assume $\frac{N\left(\mathrm{O}^{0}\right)}{N(\mathrm{O})}=0$ for $I\left(\mathrm{O}^{0}, 6300\right)=0$, $I\left(\mathrm{O}^{+}, 7320\right)>0$, and $I\left(\mathrm{O}^{++}, 5007\right)>0^{10}$.

The bottom panel of Fig. 3 shows the relative $N_{\mathrm{e}} \epsilon_{l}^{1 / 2}$ profile from $\mathrm{H}^{+}, \mathrm{He}^{+}, \mathrm{O}^{0}+\mathrm{O}^{+}+\mathrm{O}^{++}$, and $\mathrm{Ar}^{++}+\mathrm{Ar}^{3+}$ (through Eqs. (17) to (30) and assuming $T_{\mathrm{e}}=11000 \mathrm{~K}$ across the whole nebula). As expected, the ionic sequence of argon underestimates $N_{\mathrm{e}} \epsilon_{l}^{1 / 2}$ in the external, low ionization regions. Moreover, $N_{\mathrm{e}} \epsilon_{l}^{1 / 2}$ given by hydrogen is weakened by the complex de-blurring procedure, and oxygen slightly underestimates $N_{\mathrm{e}} \epsilon_{l}^{1 / 2}$ in the innermost, highest ionization layers (the same is valid for helium and argon, due to the absence of $\mathrm{He}$ II and $\mathrm{Ar} \mathrm{V}$ emissions in the spectrum).

Despite these minor discrepancies, the bottom panel of Fig. 3 accurately reconstructs the broad and asymmetrical "relative" $N_{\mathrm{e}} \epsilon_{l}^{1 / 2}$ profile along the entire true minor axis of NGC 6572; when combined with $N_{\mathrm{e}}([\mathrm{S}$ III $]$ ) (middle panel), it provides $N_{\mathrm{e}}($ top $) \simeq 21000 \mathrm{~cm}^{-3}$ (for $\left.\epsilon_{l}=1\right)$.

The matter distribution at the other PA confirms the inhomogeneous and elongated structure of NGC 6572, consisting of a dense equatorial torus and two extended and fast polar caps at lower density, embedded in a tenuous, ellipsoidal, low-excitation cocoon. The nebula is optically thick to the stellar radiation and the ionized mass, given by the observed $N_{\mathrm{e}}$ spatial distribution, the $\mathrm{H} \beta$ flux, and the radio flux (Aller 1984; Pottasch 1984; Osterbrock 1989; and Turatto et al. 2002), amounts to $M_{\text {ion }} \simeq 0.08( \pm 0.02) M_{\odot}$.

We recall that

(a) the overall spatial structure of NGC 6572 is available at http://web.pd.astro.it/sabbadin and at http://www.edpsciences.org, showing the multi-color appearance and opaque reconstruction in [O III] and [N II] (at high, medium, and low flux cuts) for a rotation around the East-West axis;

(b) further examples of "quite young" PNe ionized by a luminous star at moderate temperature, close to the thickthin transition are NGC 6210, NGC 6567, NGC 6803, NGC 6826, and NGC 6891.

\subsection{Intermediate $P N$ evolution}

In this second evolutionary snapshot, the usual, standard, double-peak +halo radial matter profile of the spherically symmetric model nebula (Table 1) is ionized by a $0.605 M_{\odot}$ hydrogen-burning post-AGB star with $T_{*}=100000 \mathrm{~K}, L_{*} / L_{\odot}=$ 6500 , and $t_{\mathrm{SW}} \simeq 4900 \mathrm{yr}$ (Blöcker 1995).

\footnotetext{
${ }^{10}$ Please note that the radial ionization structure given by Eq. (32) is independent of the nebular spatio-kinematics (i.e. expansion velocity field and matter distribution), since both the numerator and denumerator refer to the same pixel of the zvpc.
}

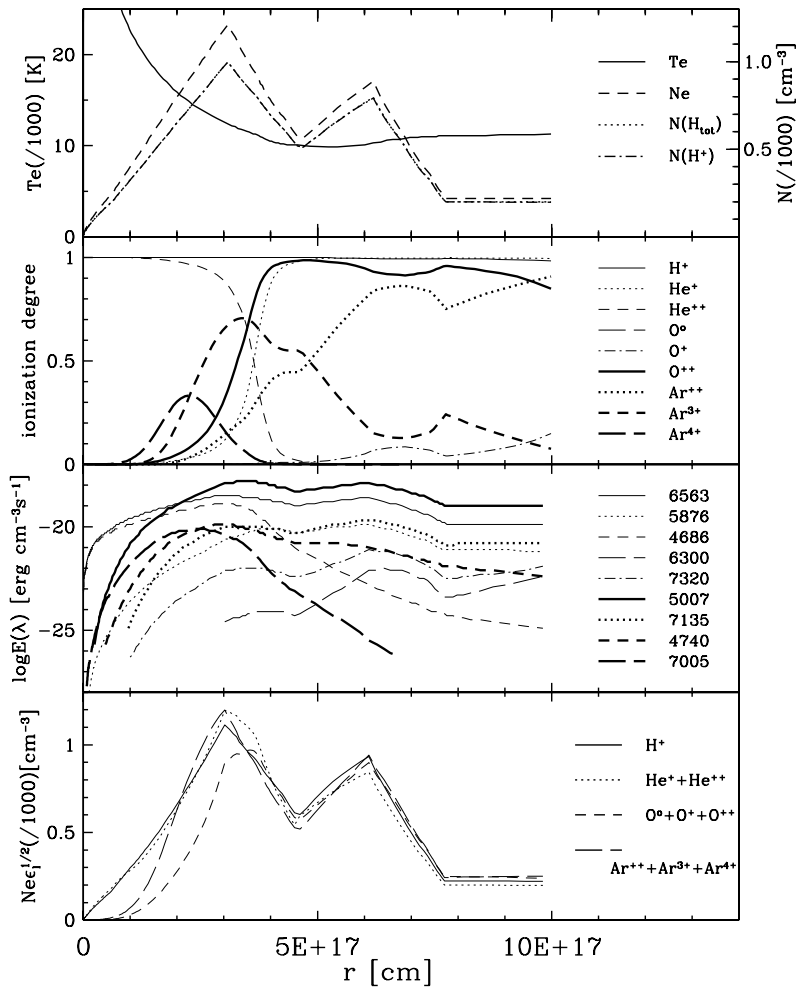

Fig. 4. Intermediate model-PN evolution (Col. 5 of Table 2). Radial characteristics of the usual, standard, spherically symmetric model nebula (Table 1 ) ionized by a $0.605 M_{\odot}$ hydrogen-burning post-AGB star with $T_{*}=100000 \mathrm{~K}$ and $L_{*} / L_{\odot}=6500$. Same symbology as Fig. 2 .

The overall properties of the model nebula are given in Col. 5 of Table 2, whereas the detailed radial characteristics (adopted density profile, physical conditions, ionization structure, and electron density profile obtained by Eqs. (17) to (30)) are shown in Fig. 4. The strength of He II and [Ar V] emissions, the absence of [O I] and weakness of [O II] together indicate that this highexcitation model-PN is decidedly thin to the UV stellar flux. The value of $T_{\mathrm{e}}$ exceeds $20000 \mathrm{~K}$ in the innermost, low-density layers, then gradually decreases to $\simeq 10000 \mathrm{~K}$ in the main regions, and increases to $\simeq 11000 \mathrm{~K}$ further.

Going to the bottom panel of Fig. 4, the $\mathrm{H} \alpha$ flux, combined with the assumption $T_{\mathrm{e}}\left(\mathrm{H}^{+}\right)=10000 \mathrm{~K}$ across the whole nebula, gives a satisfactory $N_{\mathrm{e}} \epsilon_{l}^{1 / 2}$ profile; it can be further improved adopting distinct $T_{\mathrm{e}}$ values for the inner and the outer layers. The same consideration applies to helium lines (for $T_{\mathrm{e}}\left(\mathrm{He}^{++}\right)=$ $15000 \mathrm{~K}$ and $\left.T_{\mathrm{e}}\left(\mathrm{He}^{+}\right)=10000 \mathrm{~K}\right)$.

The ionic sequence of oxygen essentially reduces to $\mathrm{O}^{++}$ (with a minor contribution of $\mathrm{O}^{+}$) and closely reproduces the external, medium excitation regions of the model nebula, but strongly underestimates the internal, high-excitation ones (adopting $\left.T_{\mathrm{e}}\left(\mathrm{O}^{+}\right)=T_{\mathrm{e}}\left(\mathrm{O}^{++}\right)=10000 \mathrm{~K}\right)$.

Argon provides a satisfactory matter distribution across the whole nebula, except in the innermost regions, where $\mathrm{Ar}^{5+}$ and even higher ionization species prevail (in Fig. 4, bottom panel, we have used $T_{\mathrm{e}}\left(\mathrm{Ar}^{4+}\right)=15000 \mathrm{~K}, T_{\mathrm{e}}\left(\mathrm{Ar}^{3+}\right)=12000 \mathrm{~K}$ and $\left.T_{\mathrm{e}}\left(\mathrm{Ar}^{++}\right)=10000 \mathrm{~K}\right)$.

From theory to practice: NGC 7009 at $\mathrm{PA}=169^{\circ}$ (apparent minor axis) is the representative example of an optically thin PN that is powered by a luminous post-AGB star at high temperature, as shown in the extensive study by Sabbadin et al. (2004), based on the absolute flux calibrated ESO NTT+EMMI echellograms at 12 PA. We refer the reader to this paper for a practi- 


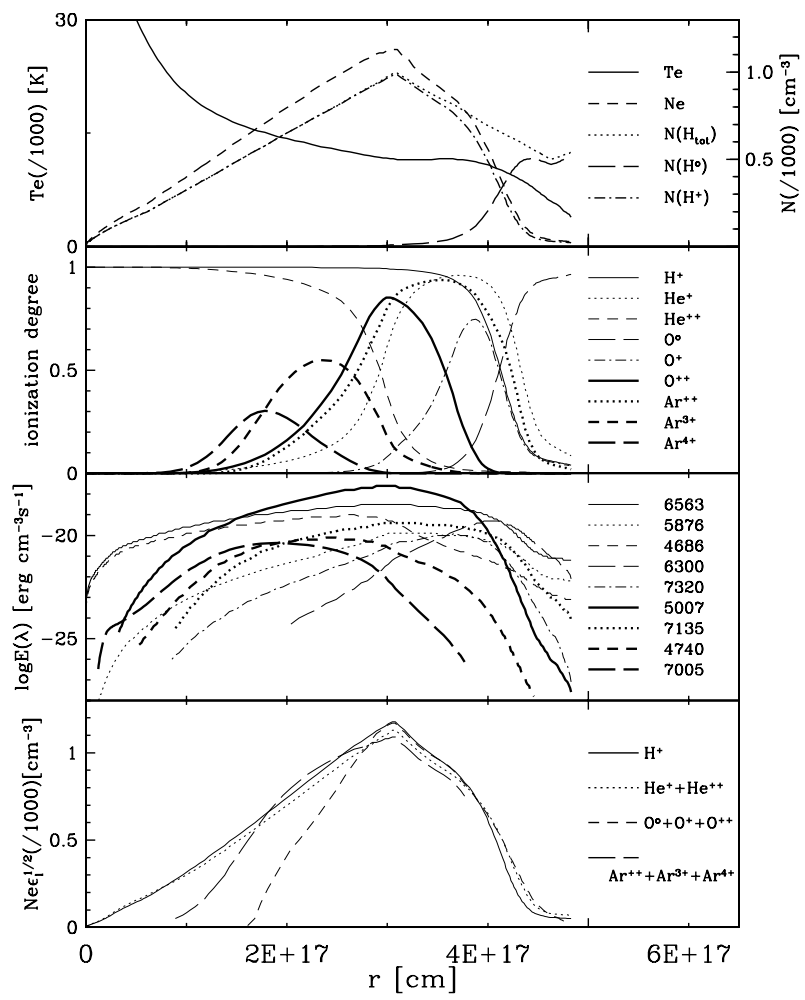

Fig. 5. Late model-PN evolution (Col. 6 of Table 2). General properties of the usual, standard, spherically symmetric model nebula (Table 1) ionized by a star with $T_{*}=150000 \mathrm{~K}$ and $L_{*} / L_{\odot}=1000$. The presence of a recombining halo is ignored by the steady-state photo-ionization code CLOUDY. Same symbols as Figs. 2 and 4.

cal application of the model-nebula discussed in this section (as well as for a detailed reconstruction of the intriguing 3D ionization structure of the Saturn Nebula).

More examples of optically thin, intermediate evolution $\mathrm{PNe}$ excited by a luminous star at high temperature are: NGC 2022, NGC 4361, NGC 6058, NGC 6804, NGC 7094, and NGC 7662.

Low-mass PNe ionized by a low-mass, slowly evolving central star become optically thinner and at higher excitation in the late evolution (thus, the considerations developed in this section remain valid), whereas "normal" and/or massive PNe powered by a "normal" and/or massive star turn to a recombination-reionization phase (as discussed in the next section).

\subsection{Late PN evolution}

According to Blöcker (1995), at time $t_{\mathrm{SW}} \simeq 7400 \mathrm{yr}$ the $0.605 M_{\odot}$ hydrogen-burning post-AGB star has $T_{*}=150000 \mathrm{~K}$ and $L_{*} / L_{\odot}=1000$; i.e. it is exhausting the hydrogen-shell nuclear burning and quickly fading along the white dwarf cooling sequence. The decreasing UV stellar flux is unable to fully ionize the nebular gas, and the outer regions recombine, thus producing a faint halo (Tylenda 1986; Phillips 2000; Turatto et al. 2002; Sabbadin et al. 2005).

Although the ionization and thermal structure of this model$\mathrm{PN}$ are out of equilibrium, the static assumption remains valid to a first approximation, since the recombination time is short: $t_{\text {rec }}=1 /\left(\alpha_{\mathrm{B}} \times N_{\mathrm{e}}\right)\left(\alpha_{\mathrm{B}}=\right.$ effective recombination coefficient $)$ is less than a century for hydrogen and even shorter for higher ionization species. Of course, the steady-state photo-ionization code CLOUDY cannot take the presence of a recombining halo into account; this it shown in Fig. 5, containing the radial characteristics of the usual, standard model nebula (Table 1), and in Column 6 of Table 2, giving the overall intensity in the main lines, the absolute $\mathrm{H} \beta$ flux, and the ionized mass.

Note (a) the very-high ionization degree of the innermost regions; (b) the strength of [O I] and [O II] emissions at the ionized edge; and (c) the temperature radial profile: $T_{\mathrm{e}}$ exceeds $30000 \mathrm{~K}$ in the innermost, low-density layers, rapidly drops to $\simeq 11500 \mathrm{~K}$ in the densest regions, and gradually decreases further ${ }^{11}$.

In Fig. 5 (bottom panel) the line-fluxes of both hydrogen (for $T_{\mathrm{e}}\left(\mathrm{H}^{+}\right)=11500 \mathrm{~K}$ ) and helium (for $T_{\mathrm{e}}\left(\mathrm{He}^{++}\right)=18000 \mathrm{~K}$ and $\left.T_{\mathrm{e}}\left(\mathrm{He}^{+}\right)=11500 \mathrm{~K}\right)$ closely reproduce the $N_{\mathrm{e}} \epsilon_{l}^{1 / 2}$ model profile. Argon (for $T_{\mathrm{e}}\left(\mathrm{Ar}^{4+}\right)=20000 \mathrm{~K}, T_{\mathrm{e}}\left(\mathrm{Ar}^{3+}\right)=15000 \mathrm{~K}$, and $\left.T_{\mathrm{e}}\left(\mathrm{Ar}^{++}\right)=11500 \mathrm{~K}\right)$ underestimates the density of the innermost layers (where $\mathrm{Ar}^{5+}$ and even higher ionization species dominate) and oxygen (for $T_{\mathrm{e}}\left(\mathrm{O}^{++}\right)=11500 \mathrm{~K}, T_{\mathrm{e}}\left(\mathrm{O}^{+}\right)=9500 \mathrm{~K}$, and $\left.T_{\mathrm{e}}\left(\mathrm{O}^{0}\right)=8000 \mathrm{~K}\right)$ only agrees in the external, medium-tolow excitation regions of the model nebula.

NGC 6565 (Turatto et al. 2002) and NGC 6741 (Sabbadin et al. 2005) are representative examples of recombining $\mathrm{PNe}$; both are high-mass objects that never became optically thin to the UV radiation of the massive, fast evolving central star. The reader is referred to these papers - based on absolute flux calibrated ESO NTT+EMMI echellograms at 6 (NGC 6565) and 9 (NGC 6741) PA - for a practical application of the model-PN developed in this section.

Following the selection criteria introduced by Sabbadin et al. (2005), further recombining PNe are: NGC 2440, NGC 2818, NGC 6302, NGC 6445, NGC 6620, NGC 6886, NGC 6894, NGC 7027, IC 4406, and Hu 1-2.

\subsection{A step backward for a special model killing two birds with one stone: proto-PNe and "born-again" low-excitation PNe}

According to the evolutionary tracks by Blöcker (1995), the combination $T_{*}=25000 \mathrm{~K}$ and $L_{*} / L_{\odot}=6500$ identifies a $0.605 M_{\odot}$ hydrogen-burning post-AGB star at time $t_{\mathrm{SW}} \simeq$ $1600 \mathrm{yr}$, corresponding to the proto-PN phase: the newly born, dense, compact, very-low excitation nebula is only partially ionized by the (still modest) UV flux of the central star. Typical examples are He 2-47, He 2-131, He 2-138, Tc 1, and M 1-46.

On the other hand, the same stellar characteristics are found in very-low excitation objects, like NGC 40 and BD $+30^{\circ} 3639$, powered by a low-temperature and high-luminosity star of late Wolf-Rayet spectral type and widely regarded as "born-again" PNe (Blöcker 1995, 2001; Herwig et al. 1999). Thus, our standard model nebula (Table 1) exposed to the radiation field of a post-AGB star with $T_{*}=25000 \mathrm{~K}$ and $L_{*} / L_{\odot}=6500$ refers to both the proto-PN phase and the low-excitation born-again PN scenario $^{12}$.

11 Points (a) and (c) explain the central hollow normally observed in the $\mathrm{H} \alpha,[\mathrm{O}$ III] and [N II] imagery of evolved PNe, like NGC 6720 (Ring Nebula) and NGC 7293 (Helix Nebula).

12 Note, however, that the collisional de-excitation of forbidden lines is important for a typical, very-high density $\left(N_{\mathrm{e}} \geq 3 \times 10^{4} \mathrm{~cm}^{-3}\right)$ protoPN. The collisional de-excitation rate is

$q_{\mathrm{de}-\mathrm{ex}}(\lambda)=8.63 \times 10^{-6}\left(\frac{\Omega}{\omega_{1}}\right) T_{\mathrm{e}}^{-0.5}$,

thus, in the high-density case, Eq. (6) becomes

$q_{\mathrm{eff}}(\lambda)=8.63 \times 10^{-6}\left(\frac{\Omega}{\omega_{1}}\right) T_{\mathrm{e}}^{-0.5} \times\left(\mathrm{e}^{\frac{-\Delta E}{k T_{\mathrm{e}}}}-1\right)$. 


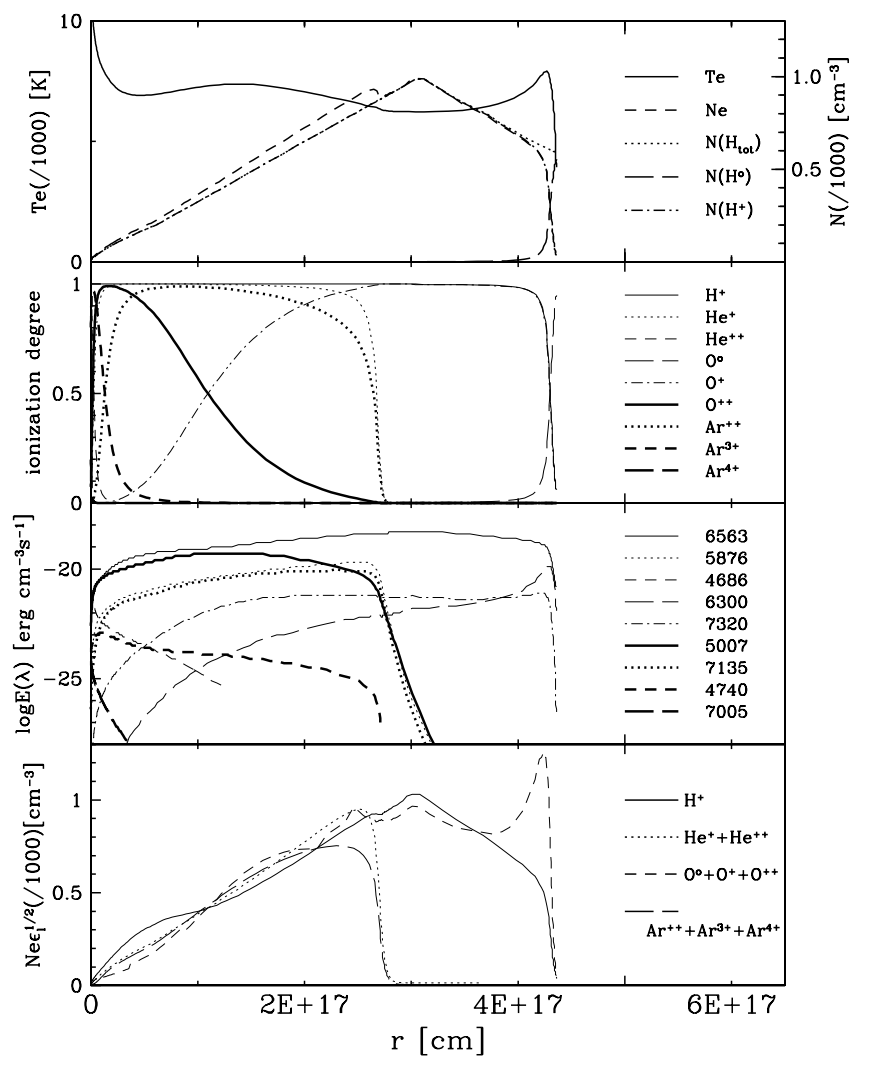

Fig. 6. Model proto-PNe + low-excitation "born-again" PNe (Col. 3 of Table 2). Radial characteristics of the usual, standard, spherically symmetric model nebula (Table 1) for an ionizing star with $T_{*}=25000 \mathrm{~K}$ and $L_{*} / L_{\odot}=6500$. Same symbols as Figs. 2, 4, and 5.

The general properties of the model nebula, summarized in Table 2 (Col. 3) and graphically shown in Fig. 6, can be synthesized as follows: the nebula is

- optically thick (very much so);

- at a very-low excitation degree (note, in particular, the weakness of $\lambda 5007 \AA$ ([O III]) and absence of He II, Ar IV, and Ar $\mathrm{V}$ emissions);

- at a moderate electron temperature: only in the innermost layers does $T_{\mathrm{e}}$ exceed $8000 \mathrm{~K}$, decreasing to $\simeq 6200 \mathrm{~K}$ in the densest regions (with an external bump up to $7900 \mathrm{~K}$ ) and declining further.

Let us analyse the $N_{\mathrm{e}} \epsilon_{l}^{1 / 2}$ profile reconstructed from line fluxes through Eqs. (17) to (30) (bottom panel of Fig. 6). A satisfactory agreement is provided by $\mathrm{H}^{+}$(assuming $T_{\mathrm{e}}\left(\mathrm{H}^{+}\right)=6200 \mathrm{~K}$ ), whereas $\mathrm{He}^{+}\left(T_{\mathrm{e}}\left(\mathrm{He}^{+}\right)=6200 \mathrm{~K}\right)$ and $\operatorname{Ar}^{++}\left(T_{\mathrm{e}}\left(\mathrm{Ar}^{++}\right)=\right.$ $6500 \mathrm{~K})$ strongly understimate the density of the main-toexternal layers at low-excitation. The ionic sequence of oxygen, $\mathrm{O}^{0}+\mathrm{O}^{+}+\mathrm{O}^{++}$, closely reproduces the matter distribution in the inner-to-main nebular regions, but presents a large discrepancy in the outermost parts (for $\left(T_{\mathrm{e}}\left(\mathrm{O}^{++}\right)=6500 \mathrm{~K}, T_{\mathrm{e}}\left(\mathrm{O}^{+}\right)=\right.$ $6200 \mathrm{~K}$, and $\left.T_{\mathrm{e}}\left(\mathrm{O}^{0}\right)=6000 \mathrm{~K}\right)$. This curious bump can be ascribed mainly to a combination of

(a) the low excitation degree of the gas $\left(\mathrm{O}^{+}\right.$is the dominant species in a large fraction of the ionized nebula), and

(b) the steep dependence of $q_{\text {coll }}(\mathrm{OII}, 7320)$ on $T_{\mathrm{e}}$ (much steeper than for the other forbidden lines of the sample).

Thus, our assumption $T_{\mathrm{e}}\left(\mathrm{O}^{+}\right)=6200 \mathrm{~K}$, constant across the $\mathrm{O}^{+}$ zone, fails in the external regions, where $T_{\mathrm{e}}$ rises to $7900 \mathrm{~K}$ (top panel of Fig. 6): it overestimates $\mathrm{N}\left(\mathrm{O}^{+}\right)$(and the corresponding $N_{\mathrm{e}}\left(\mathrm{O}^{+}\right)$contribution) by a factor of two, being $\left[q_{\text {coll }}\left(7320, T_{\mathrm{e}}=7900 \mathrm{~K}\right)\right] /\left[q_{\operatorname{coll}}\left(7320, T_{\mathrm{e}}=6200 \mathrm{~K}\right)\right] \simeq 4.3$ (whereas for the other forbidden lines of the sample $\left[q_{\text {coll }}\left(\lambda, T_{\mathrm{e}}=\right.\right.$ $7900 \mathrm{~K})] /\left[q_{\text {coll }}\left(\lambda, T_{\mathrm{e}}=6200 \mathrm{~K}\right)\right]=1.5$ to 2.3$)$.

An illustrative example of very-low excitation PN ionized by a luminous, low temperature star is $\mathrm{BD}+30^{\circ} 3639$, covered at six equally spaced PA with $\mathrm{TNG}+\mathrm{SARG}{ }^{13}$.

$\mathrm{BD}+30^{\circ} 3639$ (Campbell's hydrogen envelope star, PNG 064.7+05.0 Acker et al. 1992) is a bright $\left(\log F(\mathrm{H} \beta)=-10.03 \mathrm{erg} \mathrm{cm}^{-2} \mathrm{~s}^{-1}\right)$, high density $\left(\log N_{\mathrm{e}}=3.8\right.$ to 4.3, Barker 1978; Kingsburgh \& Barlow 1992; Aller \& Hyung 1995; Bernard-Salas et al. 2003), roundish, sharp ring $\left(5.0^{\prime \prime} \times 7.0^{\prime \prime}\right)$ surrounded by a massive envelope of neutral gas at a distance of 1200-1500 pc (Kawamura \& Masson 1996; Li et al. 2002). It is powered by a WC9 star with $m_{V}=12.5$, $T_{*} \simeq 35000 \mathrm{~K}$, and $L_{*} / L_{\odot} \simeq 4300$ (Pottasch et al. 1978; Köppen \& Tarafdar 1978; Leuenhagen et al. 1996), which is losing mass at a "moderate" wind velocity $\left(\simeq 700 \mathrm{~km} \mathrm{~s}^{-1}\right)$ and a high, although uncertain, rate (de Freitas-Pacheco et al. 1993; and Leuenhagen et al. 1996 , report $6.7 \times 10^{-6} M_{\odot} \mathrm{yr}^{-1}$ and $1.3 \times 10^{-5} M_{\odot} \mathrm{yr}^{-1}$ assuming a nebular distance of $2.0 \mathrm{Kpc}$ and $2.68 \mathrm{Kpc}$, respectively).

According to Bryce \& Mellema (1999), BD+30³639 presents a peculiar kinematical structure among $\mathrm{PNe}$, with the internal [O III] layers expanding faster than the external [N II] ones $\left(V_{\exp }([\mathrm{O} I I I])=35.5 \mathrm{~km} \mathrm{~s}^{-1}\right.$ vs. $\left.V_{\exp }([\mathrm{N} \mathrm{II}])=28.0 \mathrm{~km} \mathrm{~s}^{-1}\right)$. HST STIS observations in the C II] line at $\lambda 2327 \AA$ by Li et al. (2002) give $\left.V_{\exp }(\mathrm{C} \mathrm{II}]\right)=36.25 \mathrm{~km} \mathrm{~s}^{-1}$.

All this is confirmed and extended by our TNG+SARG echellograms. In general, they indicate that:

(a) the overall excitation degree of the gas is extremely low: mean-ionization emissions (e.g. O III, He I, and Ar III) are weak and high-ionization lines (He II, Ar IV, and Ar V) totally absent;

(b) mean-ionization species move faster than the low-ionization ones;

(c) at each PA the range in $R_{\mathrm{zvpc}}$ is quite small, suggesting a sharp radial density profile;

(d) faint, fast-expanding knots and tails ( $V_{\exp }$ up to $100 \mathrm{~km} \mathrm{~s}^{-1}$ ) are present in the strongest emissions (as already noticed by Bryce \& Mellema 1999);

(e) the spectral images are characterized by a series of local, small-scale irregularities and deformations.

In detail, the cspl vs. zvpc relation at $\mathrm{PA}=10^{\circ}$ (apparent minor axis of $\mathrm{BD}+30^{\circ} 3639$ ) based on nine ionic species enhances the presence of two distinct velocity fields: the external gas at low ionization (i.e. $\mathrm{O}^{0}, \mathrm{O}^{+}, \mathrm{S}^{+}$, and $\mathrm{N}^{+}$lines) follows the classical, Wilson's type, expansion law $V_{\text {exp }}\left(\mathrm{km} \mathrm{s}^{-1}\right)=15.0( \pm 2) \times R^{\prime \prime}$ (valid for $1.875^{\prime \prime} \leq R_{\mathrm{zvpc}} \leq 2.10^{\prime \prime}$ ), whereas the internal, higher ionization species $\left(\mathrm{O}^{++}, \mathrm{Ar}^{++}, \mathrm{He}^{+}\right.$, and $\left.\mathrm{S}^{++}\right)$move faster, but their velocity quickly decreases outwards according to the (rough) relation $V_{\exp }\left(\mathrm{km} \mathrm{s}^{-1}\right)=-43( \pm 5) \times R^{\prime \prime}+110( \pm 10)$, valid for $1.725^{\prime \prime} \leq R_{\mathrm{zvpc}} \leq 1.875^{\prime \prime}$. The contact is represented by $\mathrm{H}^{+}$, characterized by $V_{\exp }\left(\mathrm{H}^{+}\right)=28.5( \pm 1.0) \mathrm{km} \mathrm{s}^{-1}$ and $R_{\text {zvpc }}\left(\mathrm{H}^{+}\right)=1.875( \pm 0.050) \operatorname{arcsec}^{14}$.

13 The spectra of BD+30 3639 and NGC 6572 have been obtained in the same (non-photometric) night and with the same instrumental configuration. Thus, the observational information presented in Sect. 4.1 also apply to the echellograms of $\mathrm{BD}+30^{\circ} 3639$.

${ }^{14}$ In other words, $\mathrm{BD}+30^{\circ} 3639$ is a rare case of PN exhibiting the "U"-shaped expansion profile mentioned in Sect. 1. 


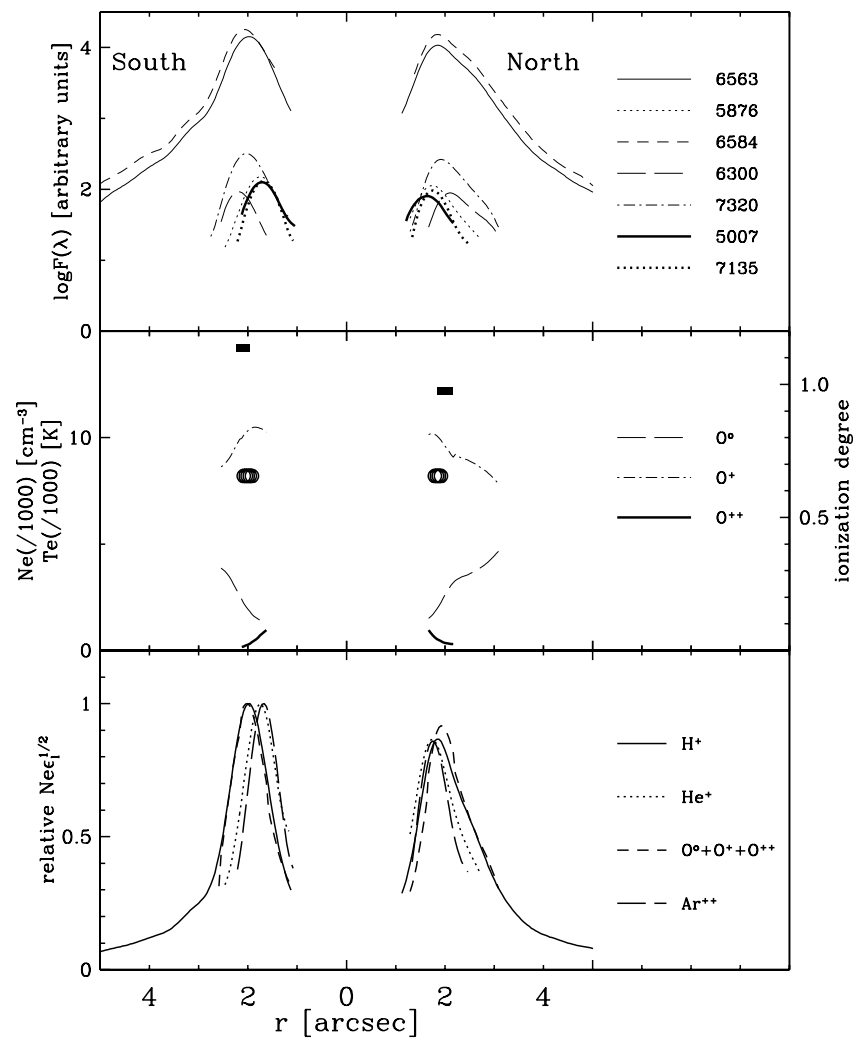

Fig. 7. Real proto-PNe and low-excitation "born-again" PNe. General properties in the $\mathrm{zvpc}$ of $\mathrm{BD}+30^{\circ} 3639$ at $\mathrm{PA}=10^{\circ}$ (apparent minor axis). Top panel: flux distribution of the main nebular emissions across the whole nebula (in arbitrary scale and corrected for $c(\mathrm{H} \beta)=0.45$ ). Middle panel: $T_{\mathrm{e}}([\mathrm{N} \mathrm{II}])$ (circles) and $N_{\mathrm{e}}([\mathrm{S} \mathrm{II}])$ (squares) at the corresponding line peak and ionization structure of oxygen (from Eq. (32)). Bottom panel: relative $N_{\mathrm{e}} \epsilon_{l}^{1 / 2}$ profile from $\mathrm{H}^{+}, \mathrm{He}^{+}, \mathrm{O}^{0}+\mathrm{O}^{+}+\mathrm{O}^{++}$, and $\mathrm{Ar}^{++}$(through Eqs. (17) to (30)). As in the case of NGC 6572 (Fig. 3), the zvpc provides two independent radial profiles of $\mathrm{BD}+30^{\circ} 3639$ in opposite directions. The central star position is at $r=0.0 \operatorname{arcsec}$, and the slit orientation is shown in the top panel. Same symbols as Fig. 3.

Of special note is that $V_{\exp } \neq A \times R^{\prime \prime}$ in the internal regions of $\mathrm{BD}+30^{\circ} 3639$; this means that the radial depth of the zvpc changes across the nebula and is given by Eq. (11), where $V_{\exp }(\lambda)$ refers to the cspl.

The general results for the $\mathrm{zvpc}$ of $\mathrm{BD}+30^{\circ} 3639$ at $\mathrm{PA}=10^{\circ}$ are shown in Fig. 7, containing the line fluxes across the whole nebula (in arbitrary scale and corrected for $c(\mathrm{H} \beta)=0.45$; top panel), the physical conditions $\left(T_{\mathrm{e}}([\mathrm{N} \mathrm{II}])\right.$ and $N_{\mathrm{e}}([\mathrm{S} \mathrm{II}])$ at the corresponding flux peak) and the ionization structure of oxygen (through Eq. (32) and assuming $\frac{N\left(\mathrm{O}^{++}\right)}{N(\mathrm{O})}=0$ for $I\left(\mathrm{O}^{0}, 6300\right)>0$, $I\left(\mathrm{O}^{+}, 7320\right)>0$, and $I\left(\mathrm{O}^{++}, 5007\right)=0$; middle panel $)$ and the relative $N_{\mathrm{e}} \epsilon_{l}^{1 / 2}$ profile (through Eqs. (17) to (30); bottom panel). As expected, $\mathrm{H} \alpha$ (for $T_{\mathrm{e}}\left(\mathrm{H}^{+}\right)=8200 \mathrm{~K}$ ) and $\mathrm{O}^{0}+\mathrm{O}^{+}+\mathrm{O}^{++}$(for $T_{\mathrm{e}}\left(\mathrm{O}^{0}\right)=8000 \mathrm{~K}, T_{\mathrm{e}}\left(\mathrm{O}^{+}\right)=8200 \mathrm{~K}$, and $\left.T_{\mathrm{e}}\left(\mathrm{O}^{++}\right)=8500 \mathrm{~K}\right)$ provide the density distribution across the whole ionized nebula, whereas $\mathrm{He}^{+}$(for $\left.T_{\mathrm{e}}\left(\mathrm{He}^{+}\right)=8500 \mathrm{~K}\right)$ and $\mathrm{Ar}^{++}\left(\right.$for $T_{\mathrm{e}}\left(\mathrm{Ar}^{++}\right)=$ $8500 \mathrm{~K})$ peak in the internal regions at higher ionization.

When combined with $N_{\mathrm{e}}([\mathrm{S} \mathrm{II}])$, the sharp matter profile in Fig. 7, bottom panel, has $N_{\mathrm{e}}$ (top) $\simeq 15000 \mathrm{~cm}^{-3}$ (for $\epsilon_{l}=1$ ) and the ionized mass of the nebula becomes $M_{\text {ion }} \simeq 0.05( \pm 0.01) M_{\odot}$.

Although a deep analysis of $\mathrm{BD}+30^{\circ} 3639$ (whose multi-color and opaque movies are shown on the WEB page http://web.pd.astro.it/sabbadin and at http://www.edpsciences.org) is beyond the aims of the paper, we wish to mention that:

- The faint halo embedding the bright nebula (see the bottom panel of Fig. 7) could be a "recombining" halo, since it essentially retains the kinematical properties observed in the external layers of the main nebula (vice versa, the kinematics of an AGB-halo is independent of the main nebula, simply reflecting the ejection law in the pre-superwind phase; for details, see Sabbadin et al. 2005). If this is the case, the past UV flux of the exciting star was much higher than the present value; less than 200 years ago, the star abruptly cooled and/or dimmed and recombination became the dominant process in the outermost nebular regions, no longer reached by stellar radiation.

- The combination of peculiar expansion velocity field + sharpness of the gas radial profile joins $\mathrm{BD}+30^{\circ} 3639$ with NGC 40, a second very-low excitation PN powered by a low temperature and high luminosity star of late Wolf-Rayet spectral type (Sabbadin et al. 2000), and identifies a small sub-class of PNe whose internal layers are probably shaped by wind interaction.

\section{Discussion}

Given a regularly expanding mass of ionized gas, the zerovelocity-pixel-column (zvpc) of high-resolution spectral images represents the tangentially moving matter at the systemic velocity and identifies a sharp radial portion of nebula in the plane of the sky, whose characteristics (density, temperature, and ionic profiles) are obtainable from line fluxes ${ }^{15}$.

The one-dimensional distribution provided by the zvpc at each PA, combined with the kinematical properties given by the central-star-pixel-line (cspl), represents the starting point for bidimensional tomography, i.e. recovery of the ionized gas structure within the entire slice of nebula intercepted by the spectrograph slit. In its turn, bi-dimensional tomography is the basis for spatial reconstruction of the whole nebula, through a 3D rendering program assembling the tomographic maps at different PA.

This is, in a nutshell, the complete logical path of our original methodology that is valid for all types of expanding nebulae, thereby overcoming any misleading camouflage due to projection and providing the true distribution of the kinematics, physical conditions, and ionic and chemical abundances at unprecedented accuracy.

In this paper we have extracted the precious information stored in the zvpc of high-resolution spectral images of PNe, focusing on the gas density profile given by different atomic species. The results for hydrogen, helium, and heavier elements can be synthesized as follows:

(I) Hydrogen (the most abundant element) presents a monoelectron atomic structure with a low ionization potential $(\mathrm{IP}=13.598 \mathrm{eV})$. At first glance, the recombination lines of $\mathrm{H} \mathrm{I}$ (whose emissivity is a weak function of the electron temperature) are excellent diagnostics of the matter distribution, since they are emitted across the whole ionized nebula. However, their effectiveness and reliability are reduced by three severe blurring sources - thermal motions, fine-structure, and expansion velocity gradient within the PN

15 Please note the affinity between the zvpc given by highresolution slit spectroscopy and the rest frame of imaging Fabry-Perot interferometry. 
- implying the accurate spectral image-reconstruction illustrated in Sect. 4.1.

(II) The same general considerations apply to the recombination lines of both ionization stages of helium.

(III) For heavier elements, we are forced to adopt collisionally excited emissions of suitable ionic sequences, because of the faintness of recombination lines, large stratification of the radiation across the nebula, and incomplete ionic coverage of the echellograms. We select $\mathrm{O}^{0}+\mathrm{O}^{+}+\mathrm{O}^{++}$for low-tomedium ionization layers (IP range from 0 to $55 \mathrm{eV}$ ) and $\mathrm{Ar}^{++}+\mathrm{Ar}^{3+}+\mathrm{Ar}^{4+}$ for the medium-to-high ionization ones (IP range from 28 to $75 \mathrm{eV}$ ). The main weaknesses of this choice (and the corresponding care) are: (1) collisional excitation rates of forbidden lines present a strong dependence on the electron temperature (in many cases, even a rough, ad hoc $T_{\text {e }}$ profile is preferable to the assumption $T_{\mathrm{e}}=$ const. across the whole nebula), and (2) ionization species higher than $\mathrm{Ar}^{4+}$ dominate in the internal regions of high-excitation PNe powered by a hot, $T_{*} \geq 100000 \mathrm{~K}$, central star $(\lambda 3425 \AA$ of [Ne V], IP range 97.1 to $126.2 \mathrm{eV}$, could be a better diagnostic for the innermost layers).

The illustrative examples presented in the previous sections show that the "best observational" density profile is always a compromise among the results given by different elements, taking the general characteristics of each target into account (evolutionary phase, optical thickness - or thinness - and excitation degree of the nebula and temperature and luminosity of the central star).

All this, combined with high-quality (i.e. $R R \geq 5$ and $\mathrm{SS} \geq 5$ ) spectroscopic material and a careful reduction procedure - thanks to the moderate dependence of $N_{\mathrm{e}}$ on the observational parameters (see Eqs. (17) to (30)) - for the first time provides accurate recovery (to within 10 to $20 \%$ ) of the radial matter distribution into an expanding, ionized mass of gas. Our zvpc analysis

(A) is based on the pixel-to-pixel measurement of velocity and flux in all nebular emissions at high, medium and low excitation contained in long-slit echellograms covering a wide spectral range. This strongly differs from (i) the usual observational setup for extended objects, inserting an interference filter to isolate a single echelle order, and (ii) the normally adopted reduction procedure, aiming either at the kinematics or at average line fluxes;

(B) is limited to the optical region (i.e. the ionized gas); for a complete understanding of the PN-phenomenology, it should be extended to other spectral domains, like near-IR (for the outermost envelope, rich in molecular and photodissociation emissions) and UV (for very-high ionization species expected in the innermost layers, where the starnebula interaction occurs);

(C) applies to other classes of expanding nebulae (e.g. nova and supernova remnants, shells around Population I Wolf-Rayet stars, nebulae ejected by symbiotic stars, bubbles surrounding early spectral-type main sequence stars, etc.) covered at adequate "relative" spatial (SS) and spectral (RR) resolutions ( $\mathrm{SS}=R / \Delta r, R=$ apparent radius, $\Delta r=$ seeing+guiding; $\mathrm{RR}=V_{\exp } / \Delta V, \Delta V=$ instrumental spectral resolution).

The zvpc methodology developed in this paper - added to tomography and 3D recovery (see Ragazzoni et al. 2001; Turatto et al. 2002; Benetti et al. 2003; Sabbadin et al. 2004, 2005) - overcomes the long-standing de-projection hurdle and opens new unusual ways to develop many facets of nebular research in more detail, in particular:

(I) to solve the topical problem of $\mathrm{PNe}$ - i.e. formation, shape, and shaping - by comparing the observational results for a representative sample of targets at different evolutionary phases with those expectations coming from current theoretical evolutionary models (Schönberner et al. 1997; Steffen et al. 1998; Marigo et al. 2001), detailed hydro-dynamical simulations (Icke et al. 1992; Frank 1994; Mellema 1997; Perinotto et al. 2004b), and updated photo-ionization codes (Ferland et al. 1998; Ercolano et al. 2003). At present, the most striking discrepancies between model-PNe (Perinotto et al. 2004b) and true-PNe (Sabbadin et al. 2005, and references therein) concern the radial distribution of

(a) kinematics: Wilson's law is reversed in model-PNe (i.e. the internal layers of the bright main shell expand faster than the medium-to-external ones);

(b) matter: in model-PNe the sharp density peak of the main shell abruptly falls inwards, whereas in true-PNe the internal decline and the whole density profile are smooth. We aim at overcoming these discrepancies (both qualitatively ascribable to an overestimation of the shaping effects due to wind interaction) by adjusting the theoretical evolutive parameters until model-PNe agree with true-PNe;

(II) to mine the nature and phenomenology of "exotic" structures at large and small scales, such as haloes, caps, ansae, jets, blobs, etc. An emblematic case is represented by the FLIERs (fast, low-ionization emitting regions) of PNe; the acronym, introduced by Balick (1978), identifies a series of external, $\mathrm{N}$-overabundant knots at low excitation and peculiar kinematics. Although the origin and evolution of FLIERs have been interpreted in many fanciful ways (instability zones at the ionization front, condensations accelerated by photo-evaporation or wind-interaction, high-speed stellar or nebular bullets, magnetically focused knots, etc.; Perinotto et al. 2004a, and references therein), our high-resolution spectral survey shows that most FLIERs are normal condensations within the outer envelope (same kinematics and chemical abundances), whose spectral characteristics can be explained fully in terms of mere photo-ionization by the central $\operatorname{star}^{16}$

(III) to disentangle the source (or sources) of general, still unsolved problems involving emission nebulae, such as the heavy-element abundance and electron temperature discrepancies obtained from optical recombination and collisionally excited lines. The proposed solutions (Peimbert et al. 2004; Liu et al. 2004; Ercolano et al. 2004; Sharpee et al. 2004, and references therein) include (a) strong temperature and density fluctuations within a chemically homogeneous nebula; (b) high-density, low-temperature knots overabundant in heavy elements embedded in a hydrogen-rich medium at lower-density and higher-temperature; and (c) other physical processes (listed in footnote 1) competing with single electron capture (i.e. recombination) in exciting high-level lines. All these (and other) hypotheses can be thoroughly tested by comparing the detailed spatio-kinematics of recombination and forbidden lines in different ions provided by the zvpc analysis, tomography, and 3D recovery applied to very-deep echellograms.

${ }^{16}$ For a deep analysis of the FLIERs, ansae and caps of NGC 7009, see Sabbadin et al. (2004). 


\section{Conclusions}

The presence of an expanding nebular gas is the typical signature of the instability phases in stellar evolution, characterized by a large, prolungated mass-loss rate (PNe, symbiotic star nebulae, shells around Wolf-Rayet and LBV (luminous blue variable) stars), or even an explosive event (nova and supernova remnants).

The mass-loss of evolved stars occupies a strategic ground between stellar and interstellar physics by raising fundamental astrophysical problems (e.g. origin and structure of winds, formation and evolution of dust, synthesis of complex molecules), by playing a decisive role in the final stages of stellar evolution, and because it is crucial for galactic enrichment in light and heavy elements.

So far, image de-projection has represented an unsourmontable obstacle to accurate recovery of the spatial structure in real nebulae, thus impeding any detailed comparative analysis with theoretical models. Such a frustrating situation is now overcome by tomography and $3 \mathrm{D}$ recovery developed at the Astronomical Observatory of Padua (Italy).

The radial density reconstruction illustrated in this paper represents the umpteenth proof that properly secured, reduced, and analysed spectra at high-resolution constitute a very useful tool for looking more closely the spatio-kinematics, physical conditions, ionic structure, and evolution of all classes of expanding nebulae, thus bridging the present, wide, and pernicious gap between model- and true-nebulae.

Acknowledgements. We wish to thank the support staff of NTT and TNG (in particular, Gloria Andreuzzi, Olivier Hainaut, Antonio Magazzú, Luca Di Fabrizio and Walter Boschin) for the excellent assistance during the observations.

F.S. greatly enjoyed general discussions with Detlef Schönberner and Robert O’Dell.

\section{References}

Acker, A., Ochsenbein, F., Stenholm, B., et al. 1992, Strasbourg-ESO Catalogue of Galactic Planetary Nebulae, ESO, Garching

Alexander, J., \& Balick, B. 1997, AJ, 114, 713

Aller, L. H. 1984, Physics of Gaseous Nebulae (Dordrecht: Reidel)

Aller, L. H. 1994, ApJ, 432, 427

Aller, L. H., \& Hyung, S. 1995, MNRAS, 276, 1101

Balick, B. 1978, AJ, 94, 671

Barker, T. 1978, ApJ, 219, 914

Benetti, S., Cappellaro, E., Ragazzoni, R., et al. 2003, A\&A, 400, 161

Bernard-Salas, J., Pottasch, S. R., Wesselius, P. R. \& Feibelman, W. A. 2003, A\&A, 406, 165

Blöcker, T. 1995, ApJ, 371, 217

Blöcker, T. 2001, Ap\&SS, 275, 1

Blöcker, T., \& Schönberner, D. 1990, A\&A, 240, L11

Bremer, M. 1995, A\&AS, 112, 551
Bryce, M., \& Mellema, G. 1999, MNRAS, 309, 731

Cerruti-Sola, M., \& Perinotto, M. 1985, ApJ, 291, 247

Corradi, R. L. M., Schönberner, D., Steffen, M., \& Perinotto, M. 2000, A\&A, 354, 1071 de Freitas Pacheco, J. A., Costa, R. D. D., de Araújo, F. X., \& Petrini, D. 1993, MNRAS, 260,401

Ercolano, B., Barlow, M. J., Storey, P. J., \& Liu, X.-W. 2003, MNRAS, 340, 1136

Ercolano, B., Wesson, R., Zhang, Y., et al. 2004, MNRAS, 354, 558

Ferland, G. J., Korista, K. T., Verner, D. A., et al. 1998, PASP, 110, 761

Frank, A. 1994, AJ, 107, 261

Gesicki, F., Acker, A., \& Zijlstra, A. A. 2003, A\&A, 400, 957

Gesicki, F., \& Zijlstra, A. A. 2000, A\&A, 358, 1058

Gratton, R., Bonanno, G., Bruno, P., et al. 2001, Exp. Astron., 12, 107

Guerrero, M. A., Villaver, E., \& Manchado, A. 1998, ApJ, 507, 889

Guerrero, M. A., Chu, Y.-H., \& Miranda, L. F. 2004, AJ, 128, 1694

Hajian, A., Terzian, Y., \& Bignell, C. 1995, AJ, 109, 2600

Herwig, F., Blöcker, T., Langen, N., \& Driebe, T. 1999, A\&A, 349, L5

Hutsemekers, D., \& Surdej, J. 1989, A\&A, 219, 237

Hyung, S., \& Aller, L. H. 1998, PASP, 110, 466

Hyung, S., Aller, L. H., \& Feibelman, W. A. 1994, MNRAS, 269, 975

Hyung, S., Aller, L. H., Feibelman, W. A., \& Lee, S. J. 2001, ApJ, 563, 889

Icke, V., Balick, B., \& Frank, A. 1992, A\&A, 253, 224

Kawamura, J., \& Masson, C. 1996, ApJ, 461, 282

Kingsburgh, R. L., \& Barlow, M. J. 1992, MNRAS, 257, 317

Köppen, J., \& Tarafdar, S. P. 1978, A\&A, 69, 363

Leuenhagen, U., Hamann, W.-R., \& Jeffery, C. S. 1996, A\&A, 312, 167

Li, J., Harrington, J. P., \& Borkowski, K. J. 2002, AJ, 123, 2676

Liu, J., Liu, X.-W., Luo, S.-G., \& Barlow, M. J. 2004, MNRAS, 353, 1231

Lucy, L. B. 1974, AJ, 79, 745

Marigo, P., Girardi, L., Groenewegen, M. A. T., \& Weiss, A. 2001, A\&A, 378, 958

Mellema, G. 1997, A\&A, 321, L29

Miranda, L. F., Vazquez, R., Corradi, R. L. M., et al. 1999, ApJ, 520, 714

Modigliani, A., Patriarchi, P., \& Perinotto, M. 1993, ApJ, 415, 258

Osterbrock, D. E. 1989, Astrophysics of Gaseous Nebulae and Active Galactic Nuclei (Mill Valley, CA Univ. Sci.)

Peimbert, M., Peimbert, A., Ruiz, M. T. \& Esteban, C. 2004, ApJS, 150, 431

Perinotto, M., Kifonidis, K., Schönberner, D., \& Marten, H. 1998, A\&A, 332, 1044

Perinotto, M., Patriarchi, P., Balick, B., \& Corradi, R. L. M. 2004a, A\&A, 422, 963

Perinotto, M., Schönberner, D., Steffen, M., \& Calonaci, C. 2004b, A\&A, 414, 993

Phillips, J. P. 2000, AJ, 119, 2332

Phillips, J. P. 2003, MNRAS, 344, 501

Pottasch, S. R. 1984, Planetary Nebulae, a Study of Late Stages of Stellar Evolution (Dordrecht: Reidel)

Pottasch, S. R., Wesselius, P. R., \& van Duinen, R. J. 1978, A\&A, 70, 629

Ragazzoni, R., Cappellaro, E., Benetti, S., et al. 2001, A\&A, 369, 1088

Richardson, W. H. 1972, J. Opt. Soc. Am., 62, 55

Sabbadin, F., Cappellaro, E., Benetti, S., et al. 2000, A\&A, 355, 688

Sabbadin, F., Turatto, M., Cappellaro, et al. 2004, A\&A, 416, 955

Sabbadin, F., Benetti, S., Cappellaro, E., et al. 2005, A\&A, 436, 549

Santander-Garcia, M., Corradi, R. L. M., Balick, B., \& Mampaso, A. 2004, A\&A, 426, 185 Schönberner, D., Steffen, M., \& Szczerba, R. 1997, Ap\&SS, 255, 459

Seaton, M. J. 1979, MNRAS, 187, 73P

Sharpee, B., Baldwin, J. A. \& Williams, R. 2004, ApJ, 615, 323

Soker, N., Zucker, D. B., \& Balick, B. 1992, AJ, 104, 215

Steffen, M., Szczerba, R., \& Schönberner, D. 1998, A\&A, 337, 149

Turatto, M., Cappellaro, E., Ragazzoni, R., et al. 2002, A\&A, 384, 1062

Tylenda, R. 1986, A\&A, 156, 217

Vassiliadis, E., \& Wood, P. R. 1994, ApJS, 92, 125

Volk, K., \& Leahy, D. A. 1993, AJ, 106, 1954

Weedman, D. W. 1968, ApJ, 153, 49

Wilson, O. C. 1950, ApJ, 111, 279

Wilson, O. C., \& Aller, L. H. 1951, ApJ, 114, 421 